\title{
DIPTERES TORRENTICOLES PEU CONNUS : I. - LES ATHERICIDAE (LARVES ET IMAGOS) DU SUD DE LA FRANCE
}

\author{
[Brachycera, Orthorrhapha].
}

par A. G. B. Thomas'.

Le nom de famille Athericidae (Stuckenberg 1973) qui sépare les Atherix sensu lato des Rhagionidae est repris. Les imagos et les larves des trois espèces du Sud de la France, Atherix ibis (Fabricius), A. marginata (Fabricius) et Atrichops crassipes (Meigen) sont décrites et comparées. L'espèce marginata est intermédiaire entre Atherix ibis et le genre Suragina Walker. Son actuel c'assement dans le genre Atherix, peu satisfaisant, est maintenu provisoirement.

La larve d'Atrichops crassipes jusqu'ici présumée aquatique est identifiće pour la première fois.

\section{Poorly known torrential Diptera : \\ I. - Larvae and adults of Athericidae (Brachycera, Orthorrhapha) from the South of France.}

\begin{abstract}
The name of the Family Athericidae (Stuckenberg 1973) which separates Atherix sensu Iato from the Rhagionidae is revived. The adults and larvae of the three species from Southern France, Atherix ibis (Fabricius), A. marginata (Fabricius) and Atrichops crassipes (Meigen) are described and compared. The species $A$. marginata is intermediate between Atherix ibis and the genus Suragina 'Walker. Its actual classification in the genus Atherix is not very satisfactory but is provisionally retained.

The larva of Atrichops crassipes was thought to be aquatic but has now been identified for the first time.
\end{abstract}

Les imagos de la famille des Rhagionidae (ou Leptidae ou Leptididae) ont fait l'objet en Europe d'un assez grand nombre de publications de systématique depuis un siècle. Dans le cadre de l'hydrobiologie, la situation est toute autre: les larves aquatiques et leurs imagos n'ont presque jamais été mentionnées, si ce n'est pour mémoire dans les travaux d'écologic ou de biologie parus jusqu'ici.

Les larves de ces Brachycères Orthorrhaphes, exclusivement prédatrices, présentent cependant un intérêt certain dans les études

1. Laboratoire d'Hydrobiologie, Université Paul-Sabatier, 118, route de Narbonne, 31077 Toulouse Cedex, France. 
de peuplements d'eaux courantes. A basse et moyenne altitude, les cours d'eau peuvent en effet héberger des Atherix, des Chrysopilus (Pomeisl 1953, Neveu 1972, nombreuses observations personnelles) et vraisemblablement les premiers stades d'au moins une espèce de Rhagio.

Malgré le petil nombre de leurs espèces franchement aquatiques, ils constituent un groupe de Diptères torrenticoles important. Ils sont d'une part très fréquents : dans les Pyrénées, il n'existe vraisemblablement pas de cours d'eau qui soil à la fois peuplé d'Éphéméroptères el dépourvu de Rhagionidae au-dessous de $1200 \mathrm{~m}$ d'altitude (les Atherix sont les plus fréquemment récoltés par les hydrobiologistes). D'autre part, la biomasse de ces larves est souvent considérable. Aldrich (1912) a signalé en Amérique du Nord des populations d'imagos si abondantes que certaines peuplades dIndiens les utilisaient comme nourriture. Plusieurs exemples de cours d'eau où ils constituent le principal groupe d'insectes prédateurs seront cités ultérieurement.

La plupart des faunes consacrées aux insectes ou aux Diptères aquatiques - ainsi que plusieurs menuels traitant des caractèrcs généraux de la biologie des eaux couranles - citent le genre Atherix (Grünberg in Braucr 1910, Johannsen 1935, Wesenberg Lund 1943, Bertrand 1954, Macan 1959, Wirth et Stone in Usinger 1963, Kauri in Illies 1967, Hynes 1970). Plusieurs auteurs mentionnent aussi ce genre dans des ouvrages relatifs à la biologic des Diptères en général, en particulier Séguy $(1950,1951,1955)$, Oldroyd (1964) el Rietschel (1969). Mais l'essentiel de nos connaissances sur la biologie des Atherix concerne le spectaculaire mode de ponte collectif d'A. ibis signalé depuis très longtemps ${ }^{1}$ (Tournier 1874, de Borre 1879, Giard 1902, etc.) et maintes fois retranscrit sans amélioration notable. Les seules contributions récentes réellement $\mathbf{u}^{\mathrm{t}} \mathrm{i}$ lisables par l'hydrobiologiste sont celles de Nagatomi (1953 à 1964) sur les espèces japonaises.

En Europe, les larves de Rhagionidac aquatiques n'ont presque pas été étudiées et les nymphes demeurent à peu près inconnues.

Je remercic M. Vaillant (Grcnoble) pour le matériel qu'il m'a apporté.

\section{LA FAMILLE DES ATHERICIDAE}

Jusqu'à Stuckenberg (1973) ont élé réunis sous le nom de Rhagionidae des Diptères de morphologic et de biologie très différentes. Par rapport aux autres Brachycères Orthorrhaphes Homéodactyles, cette famille représentait en quelque sorte « une plaque

1. Sa première description est probablement duè à Walker (1837 ?), référence incomplète citée par Brauer (1883). 
!ournante ». En réalité, les caractères de la famille restaient assez mal définis et l'ensemble manquait d'homogénéité. Quant à la division en différentes sous-familles, quelque peu variable selon les auteurs, elle était plus discutable encore. En Europe par exemple, les genres Atherix et Rhagio ont été longtemps rangés dans les Rhagioninae, alors que le genre Chrysopilus - beaucoup plus proche de Rhagio que ne l'est Atherix par la morphologie et la biologie des imagos et des larves - faisait partie des Chrysopilinae (Lindner 1925, Séguy 1926, Szilády 1932, Enderlein 1935). Celte classification artificielle était essentiellement basée sur le nombre d'épines apicales des tibias. Plus récemment, la tendance a été de classer le genre Chrysopilus dans les Rhagioninae (Leonard 1930, Szilády 1934, Frey 1954, Rozkošný el Spitzer 1965 a, Krizelj 1971). Mais lo problime de situer le genre Atherix par rapport aux autres Rhagionidae demeurait.

La proposition récente de Stuckenberg (1973) de restreindre les limites de cette famille est très séduisante. Cette étude de morphologie et d'anatomie, effectuée à l'échelle mondiale sur des imagos d'Atherix et de plusieurs genres voisins, met en évidence des relations phylétiques nettes entre ces Diptères et les Tabanidac. L'auteur propose de distinguer des Rhagionidae un petit groupe de genres en créant une nouvelle famille : les Athericidae. L'élude de la morphologie et de la biologie des larves confirme en effet la nécessité de cette séparation et le terme d'Athericidae est adopté ici.

\section{NOMENCLATURE DES ESPECES EUROPÉENNES}

\section{1. - Problèmes d'ordre spécifique.}

La présente étude porte sur trois espèces d'Atherix au sens large : A. ibis (Fabricius, 1798), A. marginata (Fabricius, 1781) ct A. crassipes: (Meigen, 1820). Il semble qu'il n'y en ait pas d'autres dans le Sud-Ouest de la France. Leur identification ne présente pas de difficultés particulières depuis le travail de Rozkošný et Spitzer (1965 a).

L'existence en Europe de quatre autres espèces d'Atherix ( $A$. apfelbecki Strobl, A. aurichalcea Becker, A. dalmatina et $A$. vicina Szilády), de diagnose assez incertaine, ne paraît pas établie de façon irréfutable : Rozkošný et Spitzer (1965 a et b) et Iscob (1972) reprennent pour l'Europe Centrale les indications de Strobl (1898), Lindner (1925) et Szilády (1932, 1934 et 1943). Une étude détaillée des genitalia ô des types semble indispensable pour éviter toute erreur due à des variations individuelles de colorations.

A titre comparatif, on trouverait en Amérique du Nord une seule espc̀ce d'après Greene (1926) et Wirth et Stone (1963); on en 
trouverait trois selon James (1965). Six espèces sont décrites du Japon (Nagatomi 1958).

\section{2. - Problèmes d'ordre générique.}

Les tableaux I et II montrent que l'espèce crassipes est sensiblement éloignée d'ibis et de marginata. Elle a été rangée par les auteurs soit dans le même genre (Atherix Meigen) que les deux autres (Grünberg 1910, Lindner 1925, Szilády 1932, Enderlein 1935) - ce qui paraît actuellement insoutenable - soit dans le genre distinct Atrichops Verrall (Séguy 1926, Szilády 1934, Rozkošný et Spitzer 1965 a, Stuckenberg 1965, Oldroyd 1969, Krizelj 1971). Au Japon, Nagatomi (1958) a scindé le genre Atherix en trois sous-genres : Atherix sensu stricto, Suragina Walker 1860 et Heterosuragina Nagatomi 1958 (= Atrichops Verrall 1909). Stuckenberg, dans une série de travaux essentiellement consacrés aux espèces australes $(1960,1966,1973)$ les érige en genres. Auparavant, deux auteurs au moins (Malloch 1932, Oldroyd 1939), avaient considéré comme très probable la synonymie Atrichops (espèce type européenne : $A$. crassipes) = Suragina (espèce type indonésienne : $S$. illucens Walker $\mathbf{1 8 6 0}$ ). De récentes études d'imagos (Nagatomi 1958, Rozkošný et Spitzer 1965 a, Stuckenberg 1965) et surtout de larves (Nagatomi 1961 a) excluent cette possibilité. Ceci est confirmé par la description, dans la présente note, de la larve d'A. crassipes inconnue jusqu'ici. La combinaison Atrichops crassipes (Meigen, 1820) est donc retenue.

Les espèces ibis et marginata ont été classées dans le même genre Atherix Meigen par les auteurs européens, excep!é Szilády (1934). En fait, leurs imagos et leurs larves présentent, à mon sens, des différences suffisantes pour justifier de leur séparation au moins en sous-gen res.

L'espèce marginata occupe en effet une position intermédiaire entre Atherix ibis et le concept du genre Suragina défini par Malloch (1932), Oldroyd (1939), Nagatomi (1958) et Stuckenberg (1960) :

- les imagos de marginata s'éloignent d'A. ibis par l'abdomen un peu plus mince, le front plus étroit chez les $q$ ( $p l$. II), les joues nettement plus étroites que le clypéus (caractères de Suragina), la tête moins globuleuse et surtout le clypéus fortement saillant et entièrement visible la!éralement; la furca génitale 오 ( $p l$. III, fig. 23) est aussi de type Suragina (comparer à Stuckenberg 1973, p. 659, fig. 18). Elles s'écartent du genre Suragina en particulier par l'absence d'éperon sur la coxa III, l'asymétric moins marquée des griffes antérieures des $\delta$, la forte pilosité (caractères d'Atherix ibis) et la coloration uniforme. 
- les larves ( $p l$. VI) diffèrent de celles d'A. ibis surtout par l'ornementation de leur revêtement et par leurs pseudopodes. Elles paraissent assez voisines de celles des deux espèces de Suragina décrites par Nagatomi (1961 a) mais s'en distinguent au moins par leurs ornementations tégumentaires épineuses, caractère qui n'a jusqu'ici été signalé ni chez Atherix ni chez Suragina.

L'une des espèces les plus proches de marginata paraît être, aux états imaginal et larvaire, Suragina kodamai Nagatomi qui appartient peut-être à un nouveau sous-genre (Nagatomi 1958).

Il me paraitrait donc souhaitable d'isoler marginata sous un nom de genre distinct mais les limites actuelles des genres Atherix et Suragina sont trop imprécises. Il existe d'autres intermédiaires que marginata entre Atherix ibis et les espèces caractéristiques de Suragina (Stuckenberg 1965), l'ensemble constituant « a remarkable morphological gradient * difficile à scinder en genres ou sous-genres.

Classer marginata dans le genre Atherix pose dans une certaine mesure le problème de la valeur même du genre Suragina. Mais en l'absence de description précise des imagos el des larves des espèces paléarctiques (autres que japonaises) el devant l'invalidité du genre Atherigia Szilády, la combinaison Atherix marginata (Fabricius, 1781), a priori peu satisfaisante, est provisoirement retenue ici.

\section{LES IMAGOS}

La but de cette étude effectuée sur 151 spécimens es! de permettre aux hydrobiologistes de distinguer les imagos d'Athericidac dans des récoltes globales de Diptères, puis de les déterminer spécifiquement sans avoir recours à une bibliographic étenduc.

Ces imagos se trouvent au voisinage de cours d'eau en général ombragés. Atherix marginata et Atrichops crassipes son! les espèces les plus représentées dans les Pyrénées, la première particulièrement en moyenne altitude. Toutes deux peuvent être assez abondantes localement mais ne constituent toujours qu'une faible partic de la totalité des Diptères récoltés aux abords des cours d'eau (Thomas 1969). Les captures d'imagos d'Atherix ibis paraissent plus rares, alors que les larves sont très répandues, et je n'ai pu obtenir les ô que par élevage.

Plusieurs critères utilisés ci-dessous sont empruntés à Séguy (1926, 1937, 1951), Nagatomi (1958), Rozkošný et Spitzer (1965 a) et Oldroyd (1969). 
1. Antennes à trois articles réellement indépendants (pl. I, fig. 1); palpes maxillaires composés de moins de 4 articles (dans le cas présent : 2) (pl. II, fig. 13) Brachycères.

2. Tête, thorax et abdomen dépourvus de grandes soies insérées dans de grandes alvéoles articulaires (macrochètes) et ne possédant qu'une fine pilosité, parfois dense; trois pelotes sub-égales sous les griffes tarsales ( $p l$. I, fig. 4) par opposition à la majorité des Brachycères qui possèdent deux pelotes latérales et un lobe central réduit à une forte soie (empodium) ( $p l$, I, fig. 5) . . . . . . . . . . Homéodactyles.

3. $3^{\text {e }}$ article antennaire réniforme avec une arista subdorsale (pl. I, fig. 1) par opposition aux Tabanidae dont le $3^{e}$ article antennaire est annelé ( $p l$. I, fig. 2 et 3 ); nervation alaire complète, épaississement costal entourant l'aile, cellule anale toujours fermée ( $p l$. I, fig. 6, 7 et 8), cuillerons thoraciques peu développés; tibia I inerme, $t$. III avec 2 éperons apicaux ............... Athericidae Stuckenberg.

Les points suivants permettent d'identifier spécifiquement les imagos des deux sexes, indépendamment des traditionnels critères basés sur les colorations :

1. Gènes glabres ( $p l$. II, fig. 14); base de la coxa I recouverte latéralement par un sclérite portant une forte denticulation antérieure ( $p l$. II, fig. 17); nervures $\mathrm{R} 4$ et $\mathrm{R} 5$ à peu près rectilignes et parallèles dans leur partie distale ( $p l$. I, fig. 8); genitalia $\hat{\delta}$ : dististyle articulé aux $3 / 4$ environ de la longueur du basistyle ( $p l$. IV, fig. 27 ); $q$ : base des cerques n'apparaissant pas nettement séparée des demi-tergites 10 très étroits (pl. III, fig. 21) ........... Atrichops crassipes (Meigen).

- Gènes nettement velues (pilosité sous-oculaire) (pl. II, fig. 12 ou 13): base de la coxa I entièrement apparente en vue latérale (pl. II, fig. 16) ; nervure R4 incurvée dans sa partie distale vers la costale et divergente de R5 ( $p l$. I, fig. 6 ou 7); genitalia of : dististyle articulé à l'extrémité du basistyle; $q$ : base des cerques apparaissant nettement séparée des demi-tergites 10 par une étroite zone claire ( $p l$. III,

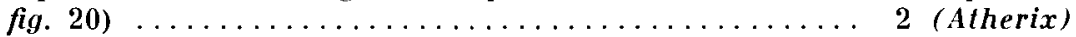

2. Bas de la face fortement déprimé, clypéus peu ou pas visible de profil $(p l$. II, fig. 12); genitalia of : pl. IV, fig. $25 \ldots \ldots \ldots \ldots \ldots \ldots \ldots$ $\ldots \ldots \ldots \ldots \ldots \ldots \ldots \ldots \ldots \ldots \ldots \ldots \ldots \ldots$ Atherix ibis (Fabricius).

- Bas de la face peu déprimé, clypéus très développé, fortement proéminent et bien visible de profll (pl. II, fig. 13); genitalia $\hat{\delta}: p l$. IV, fig. $26 \ldots \ldots \ldots \ldots \ldots \ldots \ldots \ldots \ldots \ldots$ Atherix marginata (Fabricius).

\section{Remarques :}

- Sauf spécification contraire, les descriptions qui suiven! ont été effectuées à partir de spécimens fraîchement récoltés et conservés en alcool.

- Plusieurs imagos ont été obtenues par élevage. Dans ce cas, il ne devra pas être tenu compte des da'es de métamorphose citées : ces élevages, d'une durée pouvant atteindre six mois, sont susceptibles d'avoir profondément perturbé le cycle de développement des espèces. 

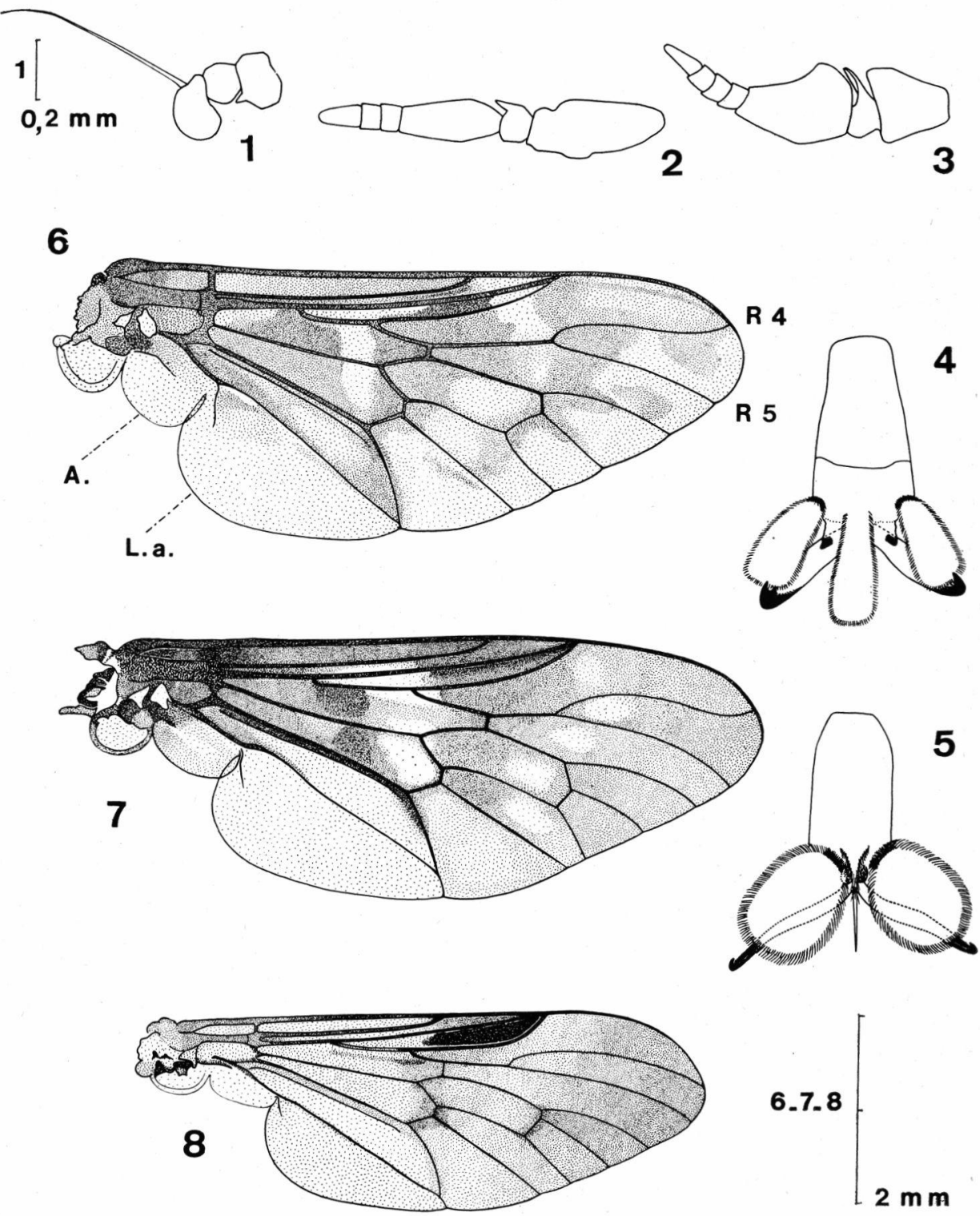

6.7 .8

$2 \mathrm{~m} \mathrm{~m}$

Planche I : fig. 1 à 8 .

- Fig. 1 à 3 : antennes gauches en vue latérale; fig. 1 : A. marginata $\uparrow$; fig. 2 et 3 : Tabanidae.

- Fig. 4 et 5 : extrémités distales de pattes postérieures droites; fig. 4 : A. ibis $q$ (homéodactyle); fig. 5: Muscidae (hétérodactyle).

- Fig. 6 à 8 : ailes droites; fig. 6: A. ibis; fig. $7:$ A. marginata; fig. 8 : A. crassipes. L. a. : lobe anal; A. : alule. 
Atherix ibis (Fabricius, 1798).

$\delta$ et $₹$ ont un aspect très différent. Les colorations paraissent assez variables.

ô - Longueur du corps : 8 à $10 \mathrm{~mm}$, de l'aile : 7 à $8 \mathrm{~mm}$.

Yeux bruns à reflets plus ou moins violacés. Tête brun noirâtre, antennes et palpes bruns, trompe brune à extrémité grisâtre. Pilosité du vertex, de la face et de la trompe noire; pilosité de la partic postérieure de la tête longue, serréc, plus fine et en grande partie blanchâtre; forte pilosité sous-oculaire noire (pl. II, fig. 12).

Thorax dorsalement brun noirâtre à pilosité noire courte et serrée, pouvant être entremêlée de poils blancs; pleures brunes à pilosité blanchâtre plus longue et plus éparse; scutellum brun noirâtre à pilosité longue et, au moins en grande partie, blanchâtre. Ailes ( $p l$. I, fig. 6) fortement maculées de brun foncé, surtout dans la partie antérieure; ptérostigma n'atteignant pas en général l'apex de la cellule marginale; nervation brun moyen; ailes se rétrécissant assez rapidement après l'aboutissement de $\mathrm{Cu}_{1}$ (largeur maximale de l'aile au niveau du lobe anal). Balanciers bruns. Coxas brun noirâtre, fémurs noirâtres (l'apex de f I et f II peul être jaunâtre). Tibia II jaunâtre, I I jaunâtre à apex brun, $t$ III élargi dans sa moitié distale et brun foncé à base jaunâtre; tarses et protarses brun foncé (la base de ces derniers peut être jaunâtre). Griffes brun jaunâtre à extrémité noire; griffe antérieurc externe nettement plus développée que les autres.

Abdomen jaune orangé vif et brun ( $p l$. III, fig. 18). Tergite 1 brun; lergites 2 à 4 inclus jaunes avec trois séries de taches brun foncé, une médiane et deux latérales; tergites suivants et genitalia bruns. Variations : les taches des tergites 2 et 4 peuvent être coalescentes et le tergite $\mathbf{5}$ en partie jaune. Ventralement, la teinte jaune est plus pâle, les taches latérales plus petites ou même absentes : dans ce cas, les sternites 2 à 4 inclus sont alors entièrement jaunes. Sternite 1 à peu près entièrement brun. Pilosité abdominale alternativement noire et dorée, correspondant grossièrement aux zones foncées et claires. Epandrium brun, parfois avcc deux laches latérales plus claires. Cerques brun foncé, aussi longs que larges ( $p l$. IV, fig. 28). Hypandrium au moins partiellement jaune. Genitalia : basistyles bruns, dististyles plus clairs; édéage fortement denté ( $p l$. IV, fig. 25).

- Longueur du corps : 8 à $11 \mathrm{~mm}$, de l'aile : 7,5 à $9,5 \mathrm{~mm}$.

Différence avec le $\delta$ : dans la pilosité sous-oculaire noire, une touffe antéricure plus claire.

Ailes moins colorées : pigment réparti plus ou moins nettemen! en bandes transversales; nervures brun plus clair; thorax brun un peu plus clair, ainsi que pleures et coxas; pattes jaunâtres à brun clair, extrémité distale des tarses plus foncée. 


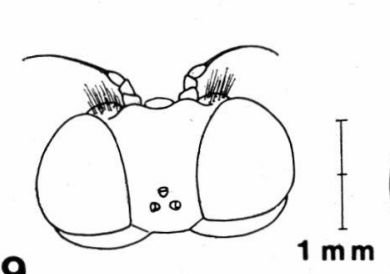

9
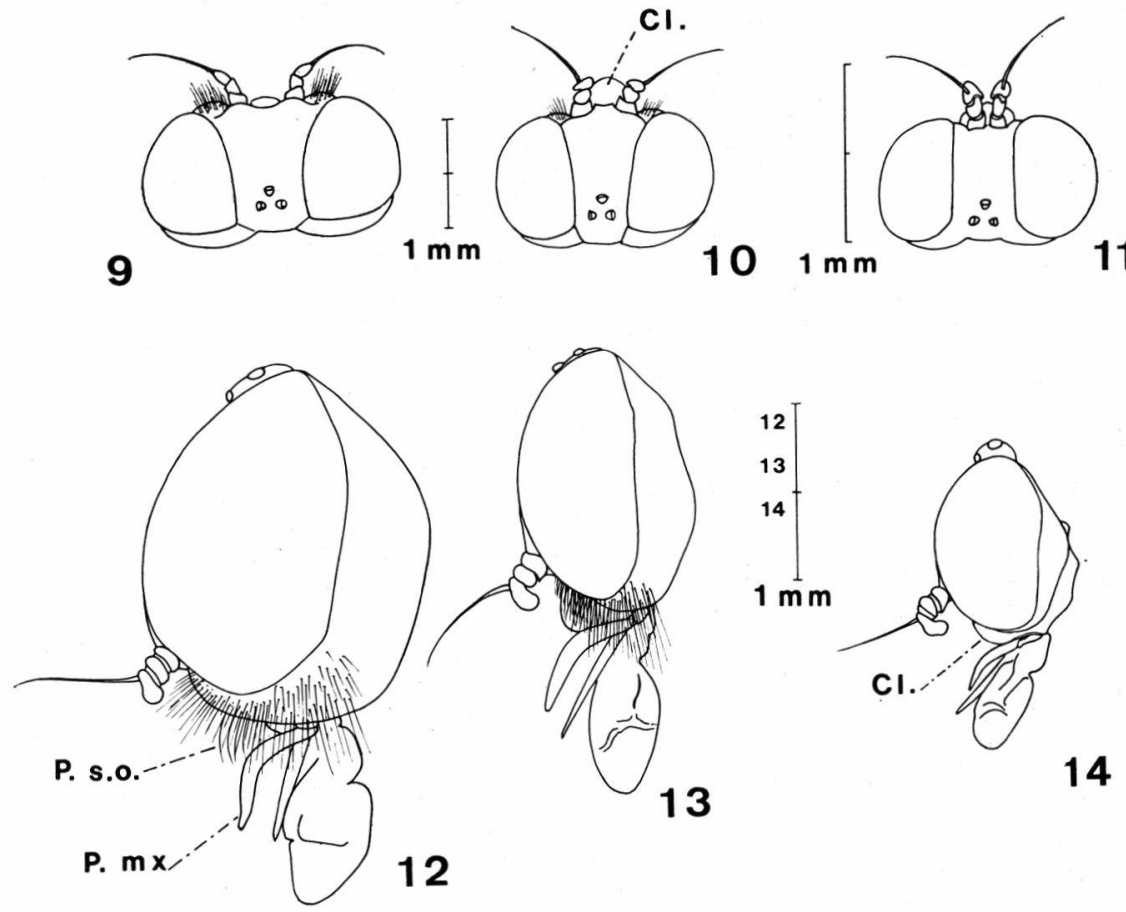

13

14

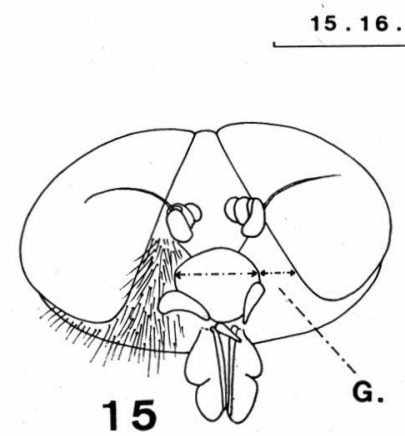

$2 \mathrm{~m} \mathrm{~m}$
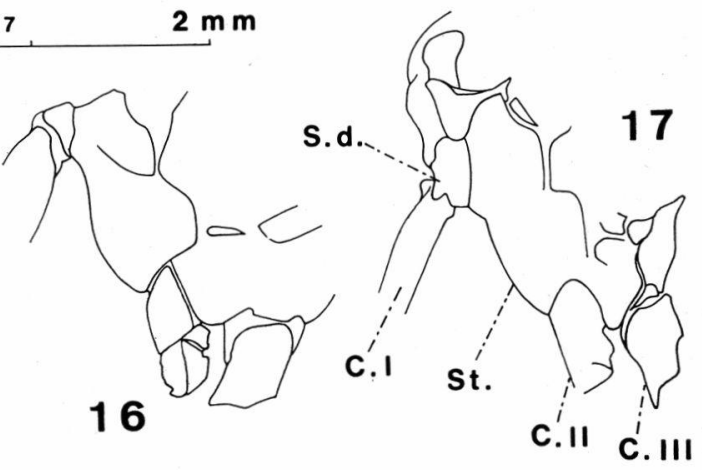

Planche II : fig. 9 à 17.

- Fig. 9 à 11 : têtes d'Athericidae + en vue dorsale; fig. 9 : A. ibis; fig. 10 : A. marginata; fig. 11 : A. crassipes.

- FIg. 12 à 14: têtes d'Athericidae $\sigma^{\text {t }}$ en vue latérale; fig. 12 : A. ibis; fig. 13 : A. marginata; fig. 14 : A. crassipes. Cl. : clypéus; P.mx. : palpe maxillaire; P.s.o. : pilosité sous-oculaire.

- FIg. 15 : tête d'A. marginata $\sigma^{\star}$ en vue ventrale. G. : gène. (FIg. 12 à 15 : seu'e la pilosité sous-oculaire a été représentée.)

- FIg. 16 et 17 : thorax d'Athericidae $\sigma^{3}$ en vue latérale; fig. 16 : A. marginata; fig. 17 : A. crassipes. S.d.: sclérite denté; St. : sternopleure; C. : coxa. 
Tergites abdominaux brun moyen; sternites plus clairs avec parfois une large auréole jaunâtre. Furca génitale ( $p l$. III, fig. 22) mince, longue et largement ajourée.

Matériel examiné : $8 \%, 22$ q.

- Hautes-Pyrénées : le déversoir du Lac de l'lle vers $2250 \mathrm{~m}, 8.8 .1968$ (8 \%, mortes antérieurement); le Nistos à $470 \mathrm{~m}, 7.8 .1968$ (7 9 , mortes antérieurement).

- Haute-Garonne : l'Escalette à $410 \mathrm{~m}, 17.7 .1973$ (1 $q$ ).

- Tarn : le Laudot à $480 \mathrm{~m}, 21$ et 25.6 .1973 (2 o obtenus gar élevage) et 12.7.1972, 24.7.1973, 5.9.1972 (2 $\uparrow, 1$ o obtenus par élevage).

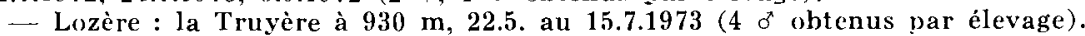

- Isère : le Furon à $980 \mathrm{~m}, 5.7 .1969$ (4 f, F. Vaillant leg.).

- Var : la Gordolasque à $1540 \mathrm{~m}, 29.5 .1967$ (1 o obtenu nar élevage, $F$. Vaillant leg.).

\section{Atherix marginata (Fabricius, 1781).}

$\hat{\delta}$ - Longueur du corps : 7,5 à $9 \mathrm{~mm}$, de l'aile : 7 à $7,5 \mathrm{~mm}$.

Différences avec $A$. ibis : yeux plus clairs à reflets variant de rouge cuivré à verl; ycux en général un peu plus espacés sur toute la hauteur du front; pilosité nettement plus longue entre ocelles et antennes; forte pilosité sous-oculaire noire ( $p l$. II, fig. 13), mais les premières soies à partir des antennes sont plus claires et inclinées vers l'arrière.

L'ensemble de la coloration de l'animal (thorax, abdcmen, pattes) varie de brun foncé assez uniforme à noir brillant selon les spécimens. Taches des ailes plus fortement pigmentées (de brun foncé à noires) ( $p l$. I, fig. 7), notamment sous le ptérostigma : celui-ci atteint l'apex de la cellule marginale mais se distingue assez mal; nervation brun foncé; en général, ailes ne se rétrécissant pas brusquement vers l'apex après l'aboutissement de $\mathrm{Cu}_{1}$. Balanciers bruns. Pates un peu plus longues et surtout plus minces que chez A. ibis. Paltes et griffes de couleur uniforme, brun foncé à noires; griffe antérieure externe nettement plus développée que les autres. Pilosité voisine de celle d'A. ibis mais plus longue ct plutôt plus foncée. Poils du thorax denses et noirs dorsalement (y compris le scutellum) clairsemés et blanchâtres sur les pleures.

Bord antérieur et partic centrale des tergites abdominaux à poils noirs, bord postérieur et parties latérales à poils clairs; sternites à poils clairs. A sec, un poudré gris, très visible sur le bord postérieur des tergites abdominaux, est à l'origine d'un aspect nettement annelé. Cerques bien plus longs que chez $A$. ibis (pl. IV, fig. 29). Genitalia : dististyle plus mince que chez cette dernière espèce; ćdéage sans dents latérales ( $p l$. IV, fig. 26).

$q$ - Longueur du corps: 9 à $10 \mathrm{~mm}$, de l'aile : 8 à $9 \mathrm{~mm}$.

Coloration assez uniforme, dans l'ensemble un peu plus claire que celle dı ô. Furca génitale ( $p l$. III, fig. 23) épaisse, courte et 

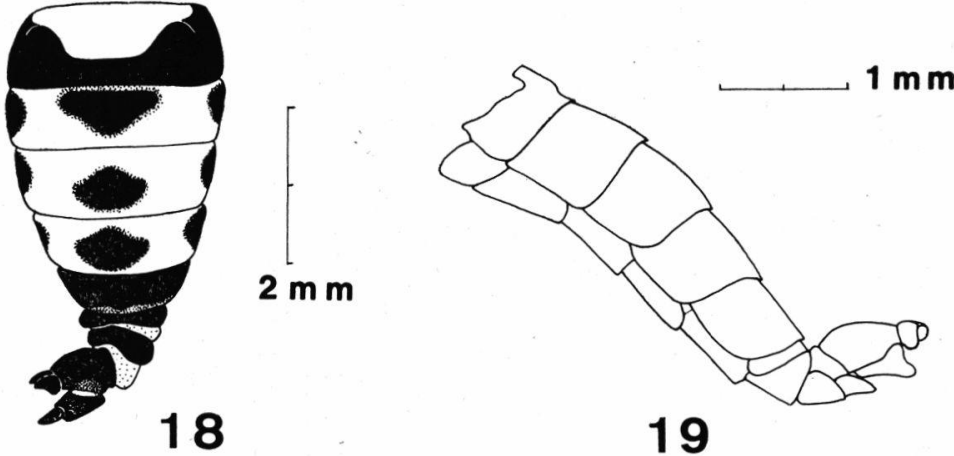

19

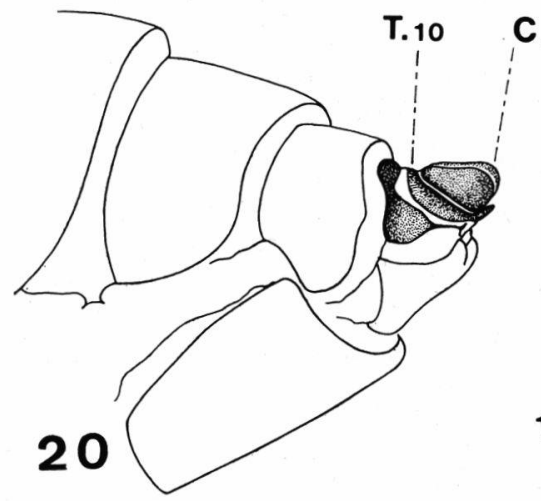

C.
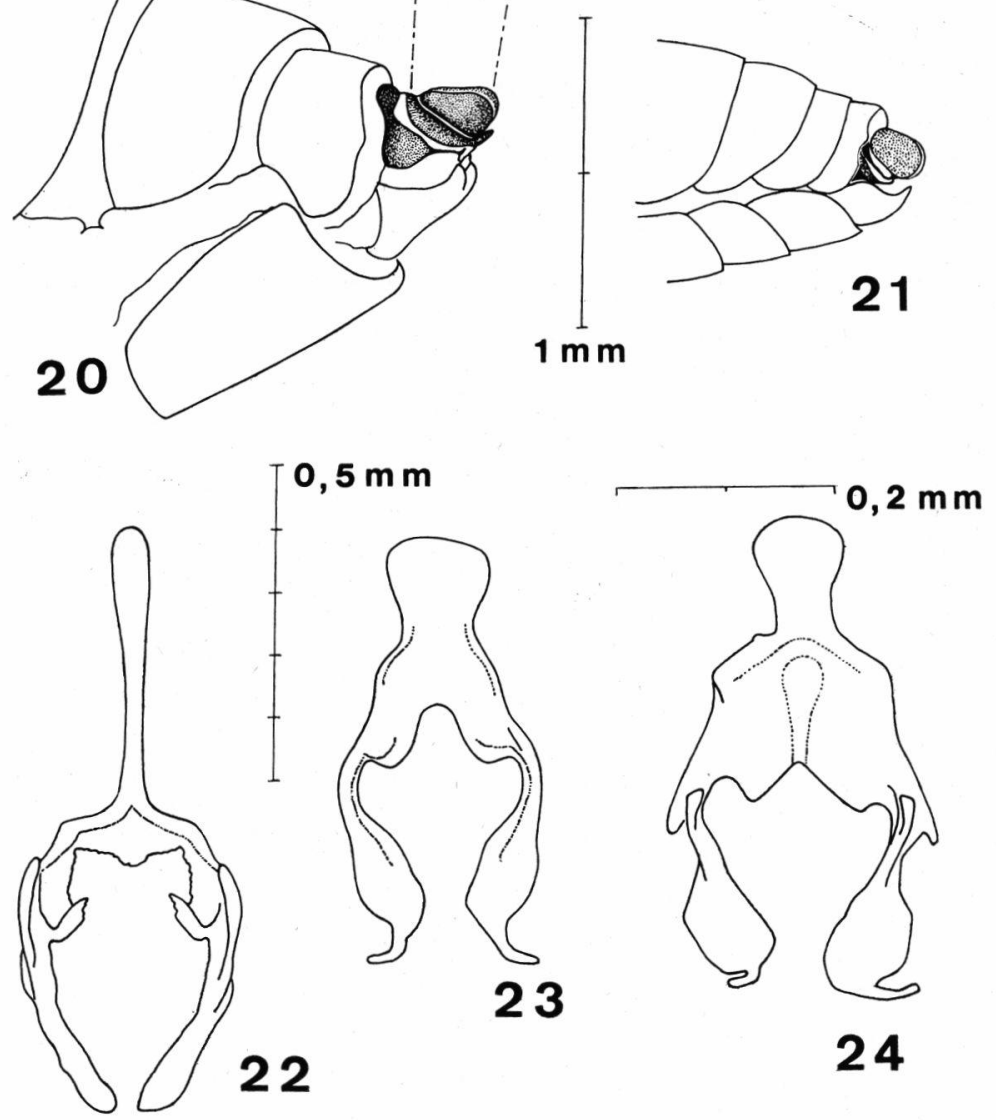

Planche III : fig. 18 à 24 .

- FIg. 18 et 19 : abdomens d'Athericidae $\sigma^{*}$; fig. 18 : A. ibis en vue dorsale; fig. 19 : A. crassipes en vue latérale.

- FIg. 20 et 21 : extrémité d'abdomens et cerques d'Athericidae $\$$ en vue latérale; fig. 20 : A. marginata; fig. 21 : A. crassipes. C. : cerque. T. 10 : 10 e tergite.

一 FIg. 22 à 24 : furcas génitales + ; fig. 22 : A. ibis; fig. $23:$ A. marginata; fig. $24:$ A. crassipes. 
relativement fermée (bien plus proche de celle d'Atrichops crassipes que de celle d'Atherix ibis).

Matériel examiné : $150^{*}, 30$ \%

- Hautes-Pyrénées : le Lavedan à $1000 \mathrm{~m}, 29.8 .1972$ ( 1 \%, morte antérieurement), 15 et 23.6.1973 ( $1 \sigma^{\circ}$ et 2 o obtenus par élevage); la Mousquère à $1150 \mathrm{~m}, 24.7 .1967\left(4 \delta^{*}, 4 \%\right)$ et $29.7 .1967(5 \%)$; la Mousquère à $1050 \mathrm{~m}$, 15.6.1973 ( 1 o obtenu par élevage); le Nistos à $470 \mathrm{~m}, 29.7 .1966$ (1 q) et 8.8.1968 (1 $\left.\delta^{*}\right)$.

- Haute-Garonne : l'Escalette à $410 \mathrm{~m}, 17.7 .1973$ (2 $\sigma^{\circ}, 6$ q); la Louge à $200 \mathrm{~m}, 18.7 .1972(1 \%)$ et 28.8 .1967 ( 1 \& obtenue par élevage); la Garonne à $140 \mathrm{~m}, 10.8 .1967$ (1 $\subsetneq$ obtenue par élevage).

- Tarn : le Laudot à $480 \mathrm{~m}, 20$ à 28.6.1973 (1 $\sigma^{\circ}, 3$ o obtenus par élevage).

- Aveyron : le Lot à $320 \mathrm{~m}, 10.7 .1973(1 \%, 1$ ㅇ).

— Lozère : le Lot à $1000 \mathrm{~m}, \mathbf{2 5 . 7 . 1 9 7 3 ~ ( 1 ~}$ o).

- Ardèche : la Glueyre à $750 \mathrm{~m}, 21.5 .1967$ (1 o obtenu par élevage, F. Vaillant leg.).

- Drôme : sources de l'Archiane vers $950 \mathrm{~m}, 6.1962$ (1 ơ, 1 q obtenus par élevage, F. Vaillant leg.).

- Isère : le Doménon à $560 \mathrm{~m}, 26.6 .1960$ (1 ó obtenu bar élevage, F. Vaillant leg.).

— Moldavie (Roumanie) : le r. Maghernița, 14.6.1967 (3 o, F. Vaillant leg.).

Atrichops crassipes (Meigen, 1820).

Grâce à M. L. Matile (Muséum de Paris) j'ai pu examiner les spécimens d'Atherix crassipes de la collection Meigen. Il s'agit bien de l'espèce citée par les auteurs européens.

Espèce nettement plus petite et plus élancée que les précédentes, en particulier le $\hat{\delta}$.

$\delta$ - Longueur du corps : 6,5 à $7 \mathrm{~mm}$, de l'aile : 5,5 à $6 \mathrm{~mm}$.

Yeux brun rouge à reflets plus ou moins cuivrés. Tête brun foncé à fort poudré gris autour des antennes, apparent même lors d'une conservation en alcool. Antennes et palpes bruns, trompe brun grisâtre. Pilosité réduite : quelques poils noirs et clairs entremêlés près des ocelles, quelques autres au-dessus des antennes; gènes glabres, pas de pilosité sous-oculaire ( $p l$. II, fig. 14); partic postérieure de la tête couverte de poils blanchâtres denses.

Thorax dorsalement brun foncé brillant à pilosité claire assez peu dense; calus huméral jaunâtre, pleures brunes et pratiquement glabres. Balanciers brun clair à tige blanchâtre. Taches des ailes assez peu distinctes, ptérostigma très visible, atteignant l'extrémité de la cellule marginale; nervation brun assez foncé. Coxas jaune clair, sauf la partie antérieure de la $3^{\circ}$ paire, brune. Fémurs I et II entic̀rement jaune clair, f III brun foncé à base jaune clair; tibias I et II jaune grisâtre, t III brun grisâtre clair; tarses bruns, partie proximale des prétarses jaune grisâtre; griffes brunes à extrémité noire. 

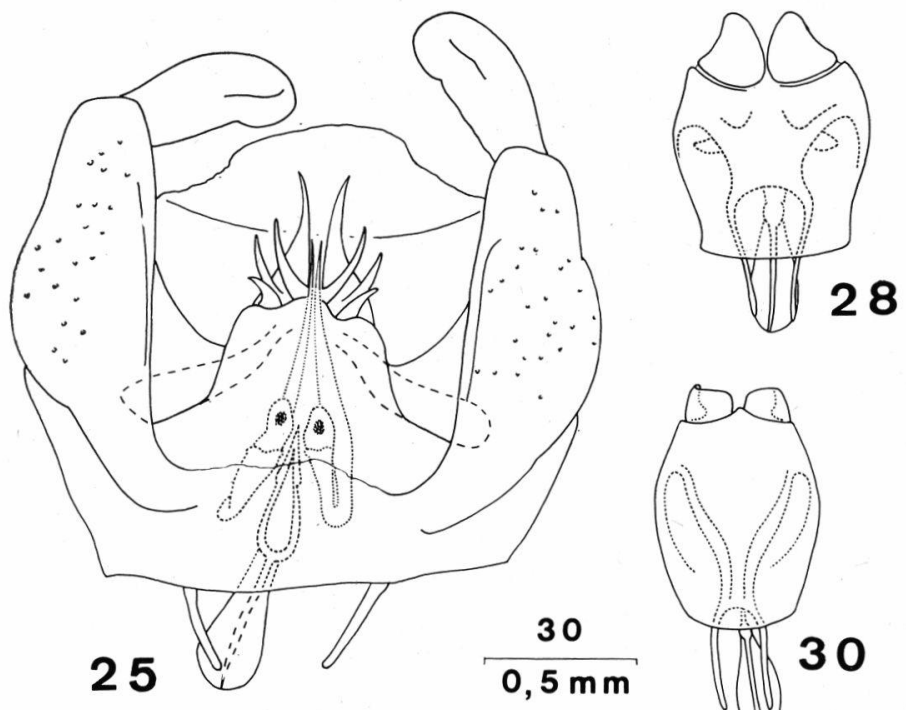

28

29

\section{$0,5 \mathrm{~m} \mathrm{~m}$}
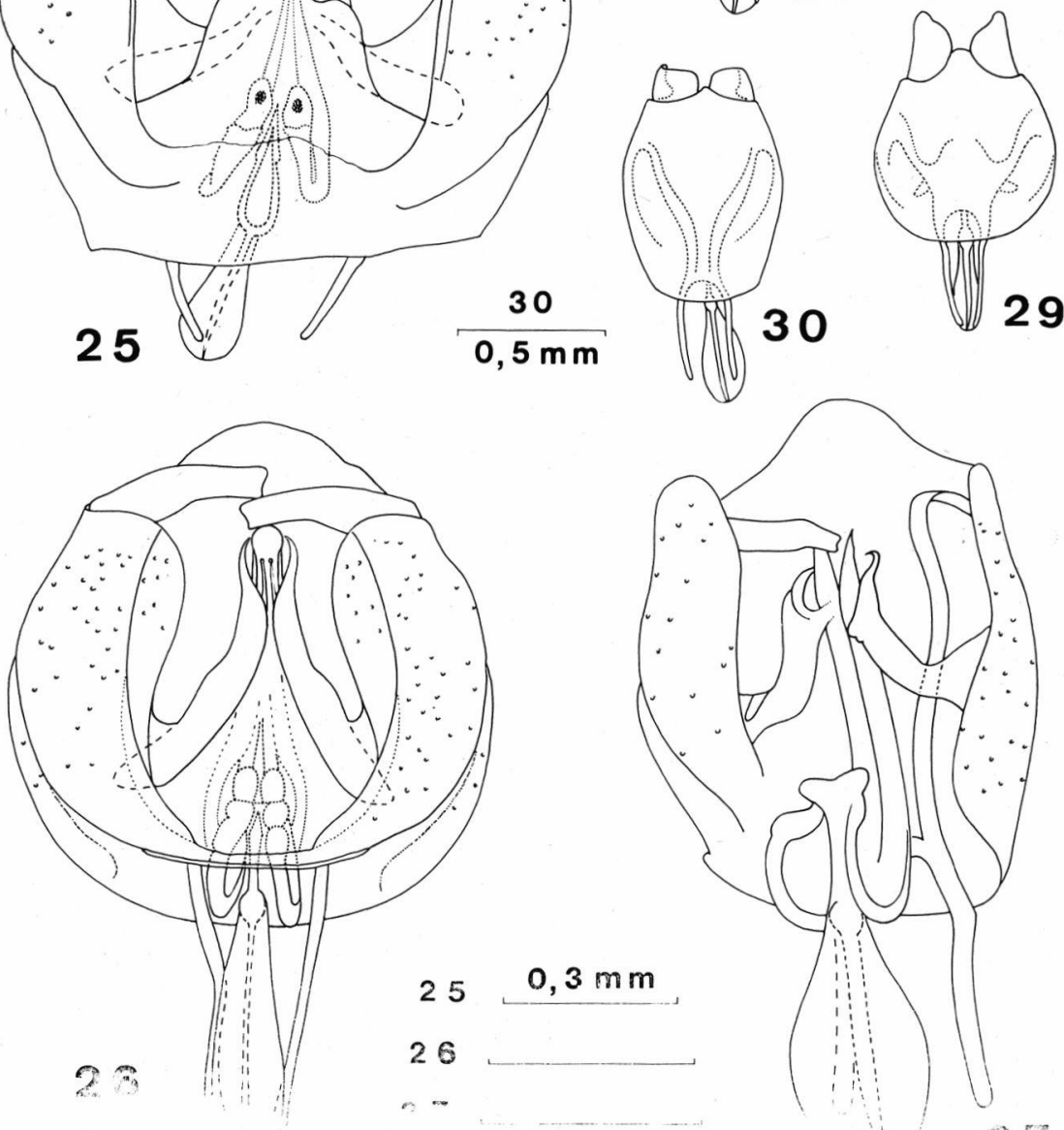

Planche IV: fig. 25 à 30 .

- Fig. 25 à 27 : genitalia $\sigma^{\star}$ d'Athericidae en vue ventrale; fig. 25 : A. ibis (préparation F. VAILlant); fig. 26:A. marginata; fig. 27: A. crassipes (style gauche enlevé).

- FIG. 28 à 30 : cerques et epandriums d'autres spécimens d'Athericidae $\sigma^{*}$ en vue dorsale; fig. 28 : A. ibis; fig 29 : A. marginata; fig. 30 : A. crassipes. 
Abdomen incurvé sur la face ventrale puis redressé de façon caractéristique ( $p l$. III, fig. 19). Tergites abdominaux brun foncé à pilosité claire et à liserés antérieur et postérieur jaune clair très marqués jusqu'au $3^{*}$ tergite inclus, moins accusés sur le $4^{*}$, à peu près inexistants ensuite : l'ensemble a un aspect annelé. Sternites 1 à 3 très clairs, presque transparents à l'exclusion d'une légère tache médiane brune (souvent claire) au bord postérieur du 2 ' sternite et plus étendue sur le $3^{*}$; sternites entièrement bruns à partir du 4". Épandrium brun foncé; cerques plus clairs, nettement plus larges que longs ( $p l$. IV, fig. 30). Hypandrium brun assez clair. Genitalia ( $l$ l. IV, fig. 27) brun foncé. Pilosité abdominale claire, plus foncée sur la partie médiane des tergites et sur les genitalia.

+ - Longueur du corps : 6,5 $\mathrm{mm}$ environ, de l'aile : $6 \mathrm{~mm}$ environ.

Coloration un peu plus claire, en particulier sur les sternites abdominaux (pas de taches sur les sternites 2 et 3 ). Furca génitale (pl. III, fig. 24) épaisse, courte et assez fermée.

Matériel examiné : 59 ơ, 17 q.

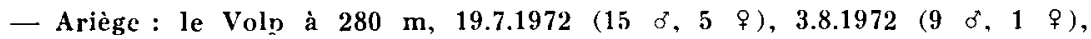
10.8.1972 ( 1 \& obtenue par élevage).

- Haute-Garonne : la Louge à $200 \mathrm{~m}, 18.7 .1972$ (10 $\delta, 2$ o), 27.7.1968 ( $\delta^{\circ}$, 1 o ), 26.7.19C7 (3 $\left.\sigma^{*}\right)$; l'Hers à $140 \mathrm{~m}, 25.6 .1934$ (2 \%, coll. A. Vandel).

- Lot, Aveyron, Lozère : le Lot entre 140 et $710 \mathrm{~m}$, du 6.7. au 7.8.1973 $\left(150^{*}, 5\right.$ \%).

- Aveyron : le Dourdou à $350 \mathrm{~m}, 5.7 .1973$ (1 q).

— Lozère : la Truyère à $930 \mathrm{~m}, 8.8 .1973\left(2 \delta^{\circ}\right)$.

- Savoie: un petit ruisseau près du lac de Saint-André vers $300 \mathrm{~m}$, 10.7.1963 (1 $\sigma^{*}, F$. Vaillant leg. $)$.

\section{LES LARVES}

La première description d'une larve d'Atherix est, à ma connaissance, celle de Dufour (1862) à partir d'un spécimen provenant de l'Adour (Landes). C'est aussi l'un des plus précises. L'auteur, qui n'en connaissait pas l'imago, l'avait classée « dans la peuplade des Muscides acalyptérés» et avait pensé pouvoir la rapporter au genre Sepedon (Tetanoceridae).

Plusieurs descriptions ou figures de larves du genre Atherix ont élé publiées depuis. Elles concernent des espèces européennes (Brauer 1883, Grünberg 1910, Wesenberg-Lund 1943), japonaises (Nagatomi 1961 a) ou américaine (Needham 1903, Greene 1926, Johannsen 1935); mais les deux seules contributions européennes d'ordre taxonomique ont été apportées par Pomeisl (1953) et Brindle (1961). Ces deux travaux traitent, le premier d'A. marginata (après élevage de larves) et d'une espèce que Pomeisl croit être A. ibis, le second d'A. ibis. Mais Pomeisl n'a pu obtenir d'imagos d'A. ibis par élevage et Brindle compare seulement la 
larve d'A. ibis à la description d'A. marginata de Pomeisl. Brindle ne précise pas si sa détermination a été effectuée à partir d'imagos obtenues par élevages de larves. Or les espèces $A$. ibis et $A$. marginata cohabitent fréquemment. Dans l'ensemble, les travaux cités sont très sommaires, à l'exception de celui de Nagatomi (1961 a).

Remarques :

- Les descriptions ci-après sont essentiellement comparatives. Elles sont basées sur des élevages qui ont permis d'obtenir des imagos des trois espèces.

- Les mesures concernent des larves mortes en extension par immersion dans l'eau chaude.

\section{Atherix ibis (Fabricius, 1798).}

Lá larve, musculeuse et active, est un redoutable prédaleur dans les écosystèmes d'eau courante. Sa longueur avant la nymphose est en général de 25 à $28 \mathrm{~mm}$ mais peut atteindre et même dépasser $32 \mathrm{~mm}$.

Le corps très hydrodynamique, netlement fuselé, comple 11 segments distincts ( $p l . \mathrm{V}, f i g .31$ et 34 ). Sternites des trois premiers dépourvus de pseudopodes. Une paire de pseudopodes par segment abdominal du premier au $7^{\circ}$ inclus, le $8^{\circ}$ portant un gros pseudopode médian unique.

Une paire d'appendices latéraux charnus souples sur les segments abdominaux 2 à 7 inclus, parfois sur le premier; dernière paire en général nettement plus longue. Une paire d'appendices dorsaux assez comparables aux précédents sur les segments abdominaux 1 ou 2 à 7 inclus, ces prolongements étant souvent réduits à de pelits tubercules jusqu'aux segments 4,5 ou 6 ; dernière paire en général plus longuc. La longueur relative de ces prolongements paraît diminuer avec la croissance de l'animal mais la variabilité est importante.

Sur le segment anal, large, les deux appendices terminaux coniques sont proportionnellement plus longs sur les larves jeunes (moins de $10 \mathrm{~mm}$ ). Ils portent deux rangées longitudinales de soies parallèles. Ces rangées sont situées dans le même plan, l'une sur la face exlerne, l'autre sur la face interne. Cette pilosité se développe au cours de la croissance de la larve : chez les larves de $8 \mathrm{~mm}$, elle est clairsemée et atleint seulement environ les $2 / 3$ oa les $3 / 4$ de la longueur du prolongement. La pilosité progresse ensuite vers l'extrémité distale des appendices et ne cesse de s'épaissir. Les soies s'aplatissent à la base et prennent la forme de rames parallc̀les dont la hauteur est orientée perpendiculairement au plan d'insertion de l'ensemble ( $p l$. V, fig. 33). Sur les larves inférieures à $3 \mathrm{~mm}$, on distingue deux soies sub-terminales, l'une externe et l'autre interne, décalées. 
La pilosité du segment anal est formée de soies latérales insérées longitudinalement en avant des précédentes. Mais elle est beaucoup plus variable pour des spécimens de même taille. De chaque côté, elle peut être fréquemment réduite à une unique soic postérieure (pl. V, fig. 32). Ultérieurement, il se forme, de chaque côté dans la partic antérieure du segment anal, une touffe de soies qui tend à rejoindre la soie postéricure à une période variable du développement. Une petite rangée de soies postérieures peut parfois se constituer indépendamment, d'où l'aspect de deux pinceaux avant leur jonction. Cette particularité signalée par Pomeisl (1953) est relativement rare; elle n'est pas caractéristique de l'espèce. L'ensemble varie beaucoup, parfois d'un côté à l'autre. Un spécimen de $25 \mathrm{~mm}$ peut ne posséder de chaque côté du segment anal qu'une seule soie postérieure. Il semble cependant que la majorité des larves de cette longueur porte une touffe bien visible de chaque côté, lout au moins dans le Sud-Ouest de la France.

Des soies unisériées sont disposées en anneaux sur les segments abdominaux, en arrière des pseudopodes à une distance de ceux-ci de l'ordre de 0,5 à 1 fois leur diamètre. Les plus visibles sont les suivantes : deux fois trois soies ventrales entre les pseudopodes, une soie plus petite derrière chaque pseudopode et une latéroventrale insérée à mi-distance entre les bases du pseudopode et du prolongement latéral. Lorsque l'animal n'est pas en parfaite extension, les six premières sont situées sur un bourrelet transversal, la dernière sur un pli longitudinal. Les soies latérales, latérodorsales et dorsales sont plus courtes et souvent peu visibles.

Sur les segments thoraciques, il y a réduction du nombre des soies mais deux soies ventrales, très développées et non ramifiées (pédichètes : pl. V, fig. 35), sont très apparentes sur chacun des trois sternites, même chez les larves de moins de $10 \mathrm{~mm}$. Celles de la troisième paire sont presque aussi longues que le premier pseudopode qu'elles précèdent. Dans la partie antérieure du premier segment thoracique, une couronne plus dense de très petites soies.

Pseudopodes de fort diamètre subdivisés - sauf ceux de la première paire et le $8^{\circ}$, unique - en deux parties, le lobe externe en général un peu plus développé que le lobe interne. L'animal s'agrippe au substrat grâce à des crochets disposés sur le tiers distal des pseudopodes en trois couronnes superposées (pl. V, fig. 38) et non deux comme l'indique Brindle (1961). Ces couronnes sont interrompues : du côté antérieur sur la première paire de pseudopodes, près de la zone longitudinale de séparation des deux lobes sur les paires 2 à 7 et du côté postérieur sur le dernier pseudopode. Les deux premiers rangs sont constitués de crochets fortement chitinisés, brun foncé, ceux de la couronne proximale étant de taille sensiblement inférieure ( $p l . \mathrm{V}, f i g .40$ et 41 ). Un troisième 

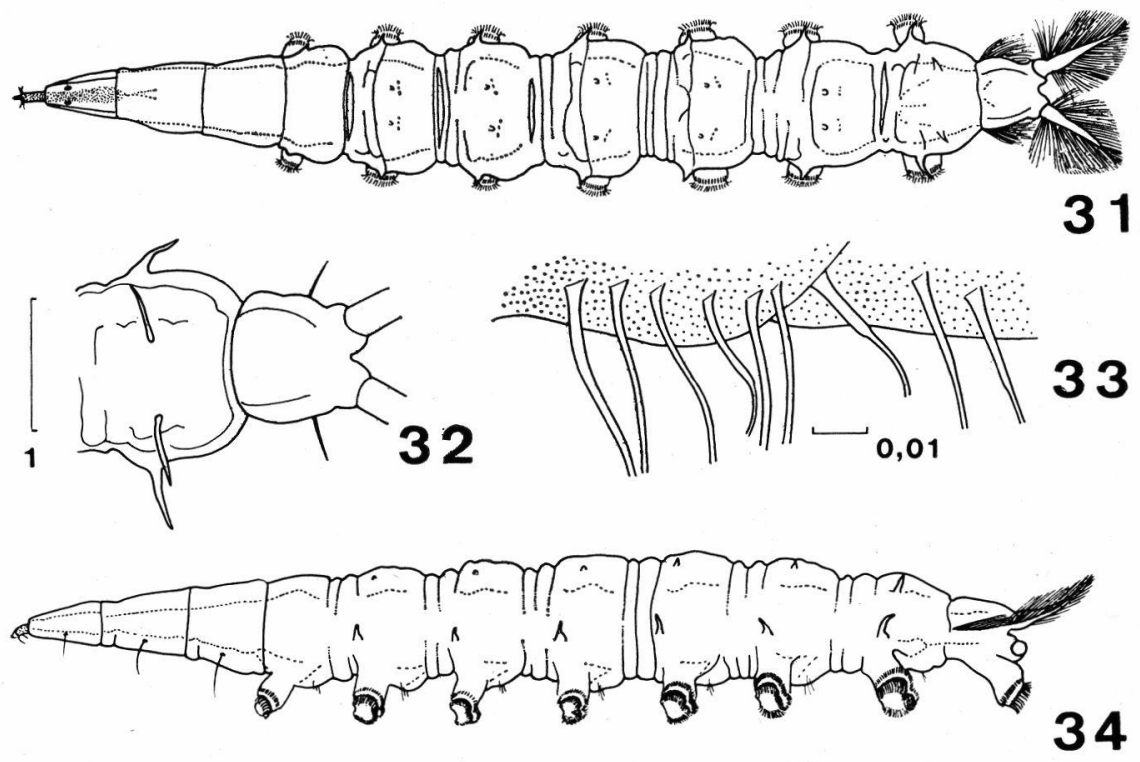

31.34 3
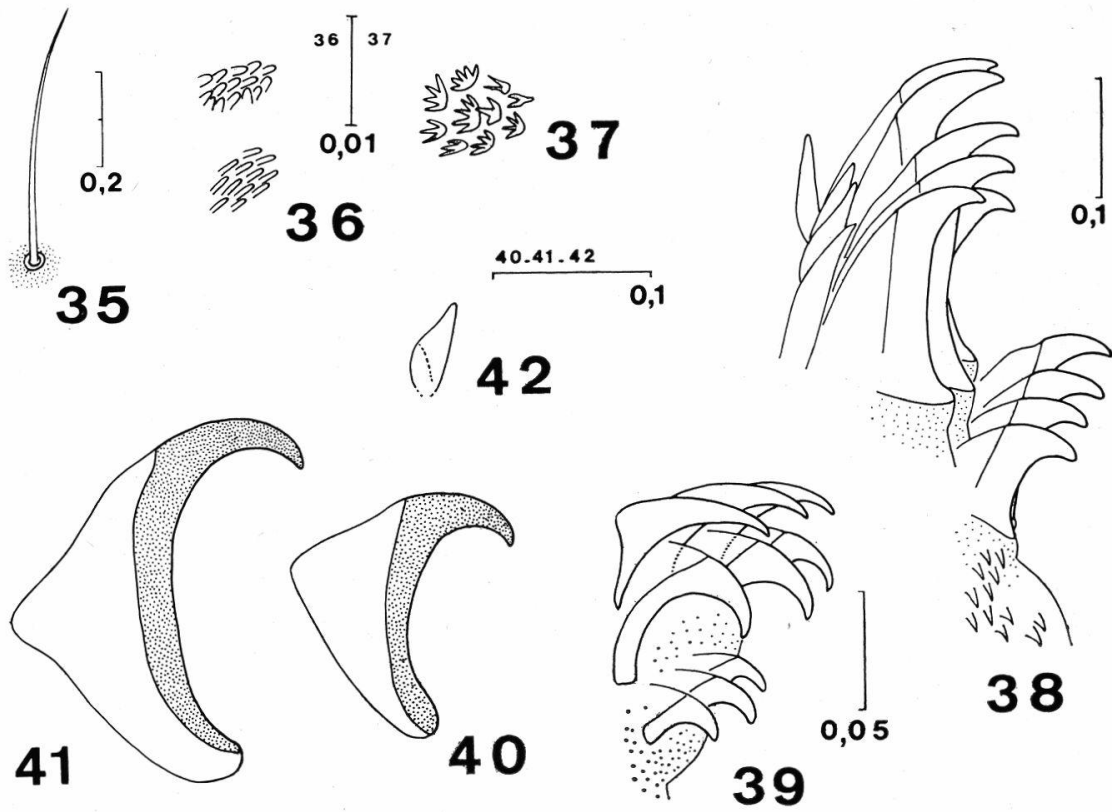

Planche V: fig. 31 à 42 (larve d'A. ibis).

- Fig. 31 : larve de $20 \mathrm{~mm}$ en vue dorsale; Fig. 32 : segment anal d'un specimen de $13 \mathrm{~mm}$; FIG. 33 : insertion des soies parallèles sur un appendice terminal; Fig. 34 : larve de $20 \mathrm{~mm}$ en vue latérale; Fig. 35 : pédichète; FIg. 36 : saillies tégumentaires abdominales dorsales; FIg. 37 : saillies tégumentaires du premier sternite thoracique; FIg. 38 et 39 : disposition des rangs de crochets sur un pseudopode $\mathrm{V}$ de larve de $22 \mathrm{~mm}$ (fig. 38) et de larve de 5,5 mm (fig. 39); FIG. 40 à 42 : crochets du rang basal (fig. 40), du second rang (fig. 41) et du rang apical (fig. 42) d'une larve de $32 \mathrm{~mm}$. Échelle en $\mathrm{mm}$. 
rang ( $p l . \mathrm{V}, f i g .42$ ), situé à l'apex du pseudopode, es! formé d'éléments moins épais à peu près rectilignes, d'autant plus courts et moins chitinisés que l’animal est âgé; sauf sur les spécimens jeunes, il ne fait pas saillie à l'extérieur de la seconde couronne. Cette régression du troisième rang est probablement due en partie à l'usure des crochets dans leur zone apicale. En effet, au-dessous d'une longueur de la larve de l'ordre de $7 \mathrm{~mm}$, les crochets du rang apical dépassent sensiblement ceux du second rang et présentent une courbure nette ( $p l . \mathrm{V}, f i g .39$ ). Le nombre des crochets du second rang s'accroît avec l'âge de la larve : sur le pseudopod: V, il est de l'ordre de 15 à 22 sur chacun des deux lobes pour une longueur de la larve comprise entre $8 \mathrm{el} 11 \mathrm{~mm}$ et de 25 à 40 entre 25 et $28 \mathrm{~mm}$ (41 sur le lobe externe el 34 sur le lobe interne chez une larve de $32 \mathrm{~mm}$ ). Premier et second rangs séparés par une distance légèrement inférieure, ou égale, à la moitié de la hauteur des crochets du premier rang. Crochets du troisième rang implanlés contre les bases transparentes des crochets du second rang, côté interne.

Zone moyenne des pseudopodes tapissée d'épines de même orientation que les crochets du premier rang ( $p l$. V, fig. 38).

La coloration, assez instable, varie de vert, plus ou moins foncś selon l'âge, à brun cuivré avec parfois alternance nel'e de zones vertes (parties convexes) et noires (partics concaves plissécs). Latéralement el ventralement, coloration plus claire; pseudopodes blanchâtres, au moins dans leur moitié distale. A un grossissement de l'ordre de 50 diamètres, le tégument paraît lisse; il es' cn réalits couvert de petites saillies arrondies très denses dont le diamètra de base est de l'ordre du micromètre ou moins pour des larves de 8 à $28 \mathrm{~mm}$ ( $p l$. V, fig. 36). Dans la partic antérieure, ces saillies s'allongent et prennent la forme d'épines (de diamc̀tre basal voi$\sin$ ), plus ou moins aiguës sur le $2^{\text {e }}$ sternite thoracique en particulier; sur le premier segment thoracique, les épines un peu plus courtes et leurs bases coalescentes confèrent à l'ensemble un aspect caractéristique, visible à faible grossissement, surlout sur le sternite ( $p l . \mathrm{V}$, fig. 37 ).

- Rapports de la longueur des appendices latéraux (a) et dorsaux (b) du $5^{\circ}$ segment abdominal à la largeur de ce segment: a : $\max .=0,48 ; \min .=0,09 ; \operatorname{moy} .=0,21 ;$ écart-type $=0,112$; coefficient de variation $=53,33 \% \quad(20$ mesures, larves de 3 à $32 \mathrm{~mm}$ ).

b : $\max .=0,10 ; \min .=0,015 ;$ moy. $=0,04 ;$ écart-type $=0,026$; coefficient de variation $=70,27 \%(10$ mesures, larves de 15 à $32 \mathrm{~mm}$ ).

- Rapport de la longueur lotale du corps à la longueur des prolongements terminaux :

$\max .=14,29 ; \min .=5,27 ; \operatorname{moy} .=10,16 ;$ écart-type $=2,055 ;$ coef- 
ficient de variation $=20,22 \%(20$ mesures, larves de 5 à $32 \mathrm{~mm})$. - Rapport de la largeur du $4^{\prime \prime}$ segment abdominal à la largeur du segment anal :

$\max .=2,04 ; \min .=1,47 ; \operatorname{moy} .=1,80 ;$ écart-type $=0,145 ;$ coefficient de variation $=8,06 \%(20$ mesures, larves de 5 à $32 \mathrm{~mm})$.

- Rapport de la longueur du pseudopode $\mathrm{V}$ à son diamètre mesuré au niveau de la couronne basale de crochets (vue latérale) : $\max .=1,48 ; \min .=1,14 ; \operatorname{moy} .=1,32 ;$ écart-type $=0,112 ;$ coefficient de variation $=8,48 \%(20$ mesures, larves de 5 à $32 \mathrm{~mm})$.

Matériel examiné : 922 larves.

- Hautes-Pyrénées : 2541.

Le ruisseau (r.) déversoir du lac de l'lle vers $2250 \mathrm{~m}$; le r. déversoir du lac Inférieur d'Estibère vers $2080 \mathrm{~m}$; le r. déversoir du lac Long à $2060 \mathrm{~m}$; le r. déversoir des méandres d'Estibère à $2040 \mathrm{~m}$; la Neste de Couplan à 1380 , 1260 et $1210 \mathrm{~m}$; le Lavedan à 1050 et $900 \mathrm{~m}$; la Neste de Louron à $900 \mathrm{~m}$; la Neste d'Aure à 660 et $490 \mathrm{~m}$; le Nistos à $470 \mathrm{~m}$.

- Haute-Garonne : 181 .

L'Escalette à $410 \mathrm{~m}$; le Volp à $240 \mathrm{~m}$; la Louge à $200 \mathrm{~m}$; la Garonne à $140 \mathrm{~m}$.

- Ariège : 921.

L'Arac à 650 et $470 \mathrm{~m}$; le Salat à $415 \mathrm{~m}$; le Volp̀ à 370 et $280 \mathrm{~m}$.

- Tarn : 3061 .

Le Laudot à $480 \mathrm{~m}$.

- Aveyron : 51 .

Le Lot à $410 \mathrm{~m}$; le Dourdou à $350 \mathrm{~m}$.

- Lozère : 2161.

La Truyère à 1210,930 et $870 \mathrm{~m}$; le Lot à 1000,710 et $550 \mathrm{~m}$.

- Isère : 21 l (F. Vaillant leg.).

Le r. de Valsenestre vers $1000 \mathrm{~m}$; le Furon à $980 \mathrm{~m}$; la Fontaine noire et le Guiers mort vers $800 \mathrm{~m}$; le Sonnant à $400 \mathrm{~m}$.

- Hautes-Alpes : 101 (F. Vaillant leg.).

Le r. de Roche Noire à $2350 \mathrm{~m}$; le r. du col du Lautaret à $2030 \mathrm{~m}$; r. près du lac Clarée vers $1850 \mathrm{~m}$; le Rif Blanc de Briançon à $1800 \mathrm{~m}$.

\section{Atherix marginata (Fabricius, 1781).}

La forme générale de cette larve rappelle bcaucoup celle d'A. ibis mais sa longueur ne paraît guère dépasser $20 \mathrm{~mm}(p l$. VI, fig. 43 et 44 ). L'espèce semble moins active; le corps est plus mou.

Les appendices latéraux sont plus longs que chez A. ibis. Brindle en fait le caractère de distinction des deux espèces. En réalité, la longucur de ces prolongements varie assez d'une larve à l'autre pour des tailles voisines mais surtout, certaines larves jeunes d'A. ibis possèdent des prolongements latéraux de grande taille et le critère peut devenir inutilisable. La longueur des prolongements dorsaux est nettement supérieure chez $A$. marginata, mais la variabilité est très forte chez $A$. ibis. Les valeurs varient dans des proportions de 1 à 6,5 pour 10 mesures seulement. Les chevauchements, quoique probablement rares, ne sont pas exclus. Le premier segment abdominal ne porte aucun prolongement. 
- Rapports de la longueur des appendices latéraux (a) et dorsaux (b) du $5^{\text {e }}$ segment abdominal à la largeur de ce segment : a : $\max .=0,98 ;$ min. $=0,30 ;$ moy. $=0,54 ;$ écart-type $=0,208$; coefficient de variation $=38,52 \%(20$ mesures, larves de 6 à $20 \mathrm{~mm})$.

b : $\max .=0,50 ; \min .=0,095 ; \operatorname{moy} .=0,30 ;$ écart-type $=0,136$; coefficient de variation $=45,33 \%$ (10 mesures, larves de 6 à $19 \mathrm{~mm}$ ).

La longueur des prolongements latéraux et dorsaux décroît au cours de la croissance chez $A$. marginata.

Même sur les spécimens en extension, la surface de l'abdomen apparaît bosselée par la présence de tubercules, plus ou moins accusés, au nombre de 12 à 18 par segment.

Le segment anal, de largeur maximale relative comparable à celle d'A. ibis, est nettement plus étroit à sa base et à la naissance des prolongements terminaux; ceux-ci sont plus grêles mais de longueur voisine. Dorsalement, une protubérance sub-terminale, absente chez $A$. ibis, avec trois soies de chaque côté. Pilosité latérale postérieure assez comparable à celle d'A. ibis mais souvent chargée de débris divers. Touffes latérales du segment anal en général bien visibles à partir de $13 \mathrm{~mm}$.

- Rapport de la longueur totale du corps à la longueur des prolongements terminaux :

$\max .=12,00 ; \min .=5,26 ;$ moy. $=8,57$; écart-type $=1,731 ;$ coefficient de variation $=20,20 \%(20$ mesures, larves de 7 à $21 \mathrm{~mm})$.

- Rapport de la largeur du $4^{\prime \prime}$ segment abdominal à la largeur du segment anal :

$\max .=2,16 ; \min .=1,67 ; \operatorname{moy} .=1,93 ;$ écart-typc $=0,134 ;$ coefficient de varialion $=6,94 \%(20$ mesures, larves de 8 à $21 \mathrm{~mm})$.

Les deux groupes de trois soies ventrales de chaque segment abdominal, plus écartés l'un de l'autre que chez $A$. ibis, rejoignent la petite soie insérée en arrière de chaque pseudopode : on dénombre donc entre les deux soies latéro-ventrales deux fois quatre soies régulièrement espacées.

Pédichètes moins robustes que chez $A$. ibis et assez fréquemment ramifiés (en général bifides) dans la moitié ou le liers distal ( $p l$. VI, fig. 45$)$.

Pseudopodes proportionnellement un peu plus longs que chez $A$. ibis mais surtout d'un diamètre plus faible et à base plus nettement conique. $\mathrm{V}$ formé par les deux lobes plus ouvert et interruption des couronnes de crochets entre les lobes plus marquée. Crochets du rang basal beaucoup plus petits, séparés du second rang par une fois leur propre hauteur environ ( $p l$. VI, fig. 47 et 48 ). Crochets du second rang ( $p l$. VI, fig. 49) un peu plus petits que chez A. ibis mais bien développés et fortement chitinisés. Crochets du troisième rang ( $p l$. VI, fig. 50) moins épais el moins recourbés 


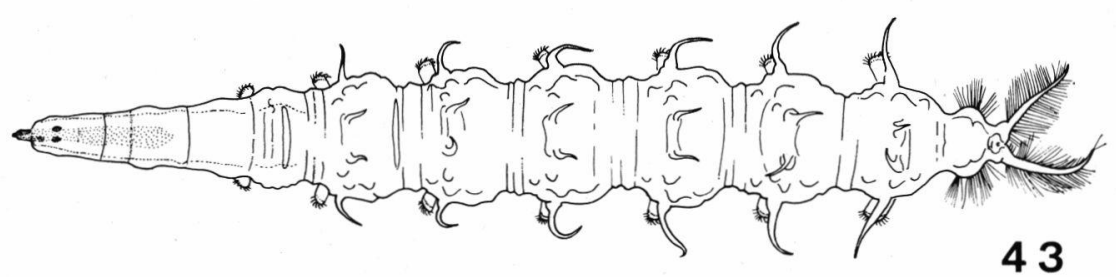

\section{$4 \mathrm{~mm}$}

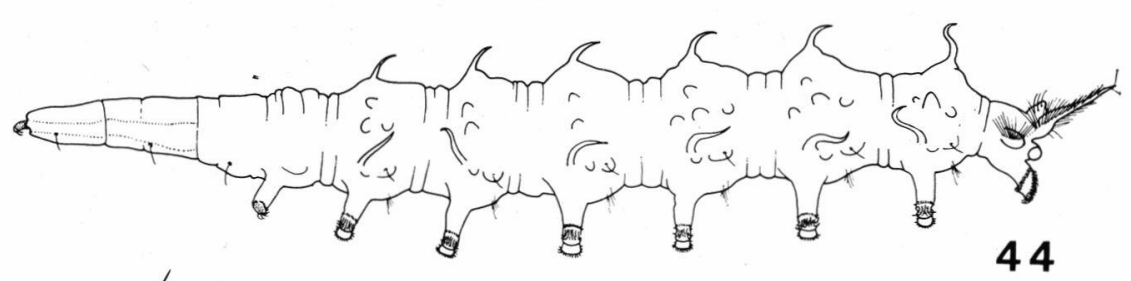

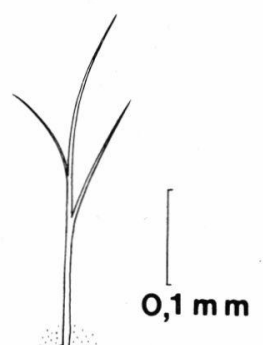

45

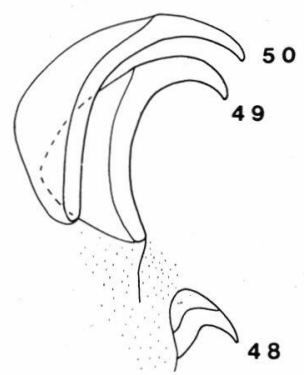

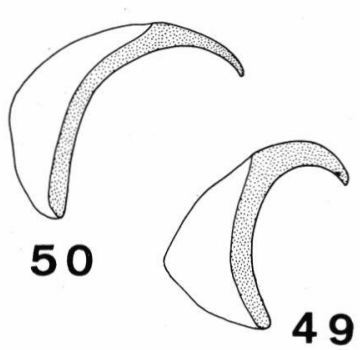

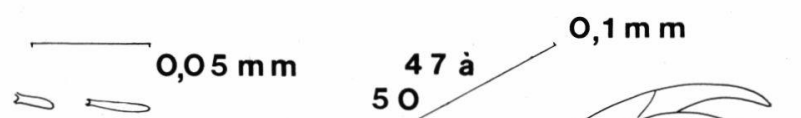

46

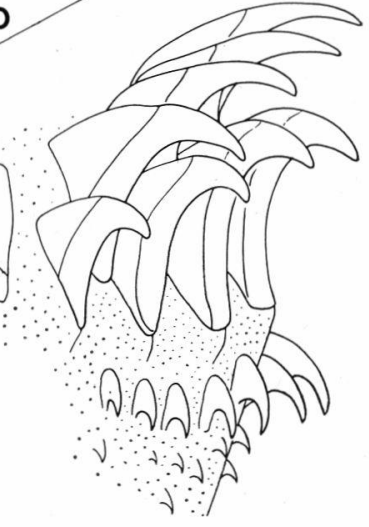

47

Planche VI : fig. 43 à 50 (larve d'A. marginata de $18 \mathrm{~mm}$ ).

- FIg. 43 : vue dorsale; FIg. 44 : vue latérale; FIg. 45 : pédichète; FIg. 46 : saillies tégumentaires abdominales dorsales; Fig. 47 : disnosition et extrémités des rangs de crochets sur un pseudopode V; Fig. 48 à 50 : crochets du rang basal (fig. 48), du second rang (fig. 49) et du rang apical (fig. 50). 
que ceux du second mais plus longs. Ils sont insérés entre les bases des crochets du second rang et sont alternés avec eux; ils les dépassent franchement en hauteur, légèrement en longueur. Contrairement à $A$. ibis, le nombre des crochets du second rang n'augmente pas au cours de la croissance de l'animal : il paraît varier (pseudopode $V$ ) de 11 à 15 par lobe pour des longueurs de larves comprises entre 9 et $20 \mathrm{~mm}$; le lobe interne compte souvent un crochet de plus que l'externe.

Epines de la zone moyenne des pseudopodes bien développées.

Coloration du corps variant de blanchâtre sale (larves jeunes) à beige parfois plus ou moins brunâtre. Sur les spécimens âgés, à un grossissement de l'ordre de 50 diamètres, on distingue aisément une multitude de petits points foncés. La surface du corps est en effet couverte de saillies en forme d'épines émoussées à divers degrés et dirigées vers l'arrière ou plus ou moins latéralement. Ces saillies sont surtout visibles sur les larves à partir de $12 \mathrm{~mm}$ environ ( $p l$. VI, fig. 46); leur largeur à la base est de l'ordre de $5 \mu \mathrm{m}$ ou plus chez les larves âgées $(15$ à $20 \mathrm{~mm})$, nettement moins chez les spécimens plus petits. Elles sont particulièrement développées sur la partie médiane des tergites abdominaux autour des prolongements dorsaux. Elles constituent le meilleur critère de distinction entre $A$. marginata et $A$. ibis lorsqu'il s'agit de spécimens con!ractés, à pseudopodes non apparents.

- Rapport de la longueur du pseudopode $\mathrm{V}$ à son diamètre mesuré au niveau de la couronne basale de crochets (vue latérale) : $\max .=3,12 ; \min .=2,23 ; \operatorname{moy} .=2,63 ;$ écart-type $=0,221$; coefficient de variation $=8,33 \%(20$ mesures, larves de 8 à $21 \mathrm{~mm})$.

Matériel examiné : 316 larves.

- Hautes-Pyrénées : 1901.

La Neste de Couplan à 1380 et $1210 \mathrm{~m}$; l'Espiaube à 1280,900 et $810 \mathrm{~m}$; la Mousquère de 1250 à $1050 \mathrm{~m}$; le Lavedan à 1050 et $900 \mathrm{~m}$; le $\mathrm{r}$. de Lastie à $1100 \mathrm{~m}$; le r. d'A rdengost à 1150 et $1000 \mathrm{~m}$; le r. de Beyrède à $1000 \mathrm{~m}$;

le Baricave à $700 \mathrm{~m}$; le r. de Sarranet à $690 \mathrm{~m}$; le Nistos à 600 et $470 \mathrm{~m}$; le

r. de Sauveterre à $470 \mathrm{~m}$.

-C Haute-Garonne : 18 l.

L'Escalette à $410 \mathrm{~m}$; le Volp à $240 \mathrm{~m}$; la Louge à $200 \mathrm{~m}$; le r. de Réganel à $190 \mathrm{~m}$; l'Ariège à $150 \mathrm{~m}$; la Garonne à $140 \mathrm{~m}$.

- Ariège : 241.

Le r. des Cors à $830 \mathrm{~m}$; l'Arac à $650 \mathrm{~m}$; le Baup à 520 et $400 \mathrm{~m}$; le r. de Perri à $410 \mathrm{~m}$; le Volp à 430 et $280 \mathrm{~m}$.

- Aude : 1 l.

Le r. d'Encouloum à $360 \mathrm{~m}$.

- Tarn : 521 .

Le $r$. de Laudot à $480 \mathrm{~m}$.

- Aveyron : 21.

Le Dourdou à 350 et $230 \mathrm{~m}$.

- Lozère : 161 .

Le Lot à 1130 et $1000 \mathrm{~m}$; la Truyère à $930 \mathrm{~m}$.

-- Ardèche : 1 l. (F. Vaillant leg.).

Le $r$. de la Violle vers $800 \mathrm{~m}$.

- Isère : 12 l. (F. Vaillant leg.).

Le Furon à $980 \mathrm{~m}$; le Bruyant à $980 \mathrm{~m}$; le Doménon à $560 \mathrm{~m}$; le Vernon à $400 \mathrm{~m}$. 


\section{Atrichops crassipes (Meigen, 1820).}

Aspect général ( $p l$. VII, fig. $51 \mathrm{~A}$ et 52) très différent de celui des espèces précédentes. La longucur ne paraît pas excéder $15 \mathrm{~mm}$.

Quatre prolongements (deux dorsaux et deux latéraux) sur les segments abdominaux 6 et 7 , deux terminaux sur le segment anal. Ces dix prolongements très longs, grêles et dirigés vers l'arričre, sont de dimensions assez voisines. Les six derniers sont toutefois plus longs. Ces appendices peuvent accidentellement être de longueur réduite et terminés par une cicatrice noirâtre. Les prolongements terminaux ne portent pas de soies parallèles.

- Rapport de la longueur totale du corps à la longueur des prolongements terminaux :

$\max .=3,57 ; \min .=2,70 ;$ moy. $=3,14$; écart-type $=0,230 ;$ coefficient de variation $=7,32 \% \%^{\circ}(20$ mesures, larves de 6,5 à $14,5 \mathrm{~mm})$.

Sur chaque segment abdominal 2 à 5 inclus, au-dessus des pseudopodes, deux fortes protubérances dorsales coniques ou plus ou moins pyramidales de hauteur et de diamètre basal comparables; latéralement, deux protubérances de chaque côté, d'aspect identiquc, trìs rapprochées et supcrposées.

- Rapports de la hauteur des protubérances latérales (a) et dorsales (b) dı $5^{*}$ segment abdominal à la largeur de ce segrient : a : $\max .=0,15 ; \min .=0,06 ; \operatorname{moy} .=0,10 ; \quad$ écart-type $=0,028$; coefficient de variation $=27,96 \%$ (10 mesures, larves de 6,5 à $14,5 \mathrm{~mm}$ ).

$\mathrm{b}: \max .=0,17 ; \min .=0,08 ; \operatorname{moy} .=0,11 ;$ écart-lype $=0,027$; coefficien! do variation $=25,84 \%(10$ mesures, larves de 6,5 à $14,5 \mathrm{~mm}$ ).

La surface de l'abdomen porte en outre de nombreuses constrictions et des crêtes transversales moins visibles.

Le segment anal, de taille réduite et fortement rétréci à la base, ne porte pas de soies latérales parallèles. Dorsalement, en avant de la naissance des prolongements terminaux, une forte protubérance sub-terminale, proportionnellement plus marquée que chez A. marginata.

Celte protubérance porte de chaque côté, à mi-hauteur, une soie noire et non trois comme chez $A$. marginata.

- Rapport de la largeur du 4" segment abdominal à la largeur du segment anal :

$\max .=3,45 ; \min .=2,56 ;$ moy. $=2,93 ;$ écart-type $=0,356 ;$ coefficient de variation $=12,15 \%(10$ mesures, larves de 6,5 à $14,5 \mathrm{~mm})$.

Sur chaque segment abdominal, entre les pscudopodes et en arrière de ceux-ci, de chaque côlé, un groupe de trois soies noires rapprochées sur une légère protubérance; soie latéro-ventrale noire bien visible. 
Pédichètes ramifiés en 3 ou 4 branches, en général dès la basc (pl. VII, fig. 53). Sur le reste du corps, soies fréquemment ramifiées, surtout dans la partie antérieure.

Une paire de pseudopodes bilobés - sauf ceux de la première sur les segments abdominaux 1 à 7 inclus (pas de pseudopode sur lc segment anal). 7 " paire de taille inférieure.

- Rapport de la longueur du pseudopode $V$ à son diamìtre mesuré au niveau de la couronne basale de crochets (vue latérale) : $\max .=3,20 ; \min .=2,44 ; \operatorname{moy} .=2,87 ;$ écart-type $=0,260 ;$ coefficient de variation $=9,06 \%$ (10 mesures, larves de 8 à $14,5 \mathrm{~mm})$.

$V$ formé par les deux lobes encore plus ouvert que chez $A$. marginata. Les pseudopodes des deux espèces sont de morphologie assez voisine. On relève cependant quelques différences.

Les crochets du rang basal ( $p l$. VII, fig. 56) sont proportionnellement plus gros que chez $A$. marginata, plus espacés et en nombre très réduit (en général 6 à 8 soit 4 à 5 fois moins nombreux). Ce premier rang est très rapproché du second ( $p l$. VII, fig. 55 et 57) qui s'insère presque immédiatement au-dessus et en quinconce. Crochets du troisième rang ( $p l$. VII, fig. 58) plus grands el plus ouverts que ceux du second, aussi épais, insérés entre les crochets de ce dernier mais à peu près à leur sommet : interpénétration moins marquée que chez $A$. marginata. Aux extrémités de chaque rang, le dernier crochet est de taille réduite, les rangs se rapprochent les uns des autres et peuvent se distinguer assez mal. Le

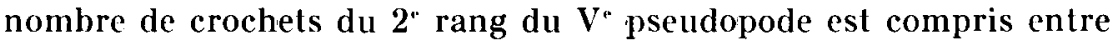
6 et 9 (lobe externe seulement) pour des longueurs totales de larves de 8 à $14,5 \mathrm{~mm}$.

Zone moyenne des pseudopodes couverte d'épines de même orientation que les crochets du premier rang.

Le rang apical de crochets est de plus en plus développé dans la séric: A. ibis, A. marginata, A. crassipes.

La coloration de l'animal varie de beige grisâtre à marron, parfois plus ou moins orangée, selon le milieu où il vit. Les larves sont fréquemment très sales, couvertes de débris retenus par de fins prolongements, parfois très longs, plus ou moins flexueux et souvent serrés ( $p l$. VII, fig. 54). Leur densité est plus grande dans la région postérieure du corps, près de la base des appendices dorsaux et latéraux. Elle semble varier d'un cours d'eau à l'autre.

La larve d'A. crassipes paraît voisine de celle d'A. fontinalis (Nagatomi, 1958) décrite par Nagatomi (1961 a).

Matériel examiné : 52 larves.

- Ariège : $19 \mathrm{l}$,

Le Volp à 290 et $280 \mathrm{~m}$.

- Haute-Garonne : 161.

Le Volp à $240 \mathrm{~m}$; la Louge à $200 \mathrm{~m}$; la Garonne à $140 \mathrm{~m}$.

- Lot-et-Garonne : 21. 

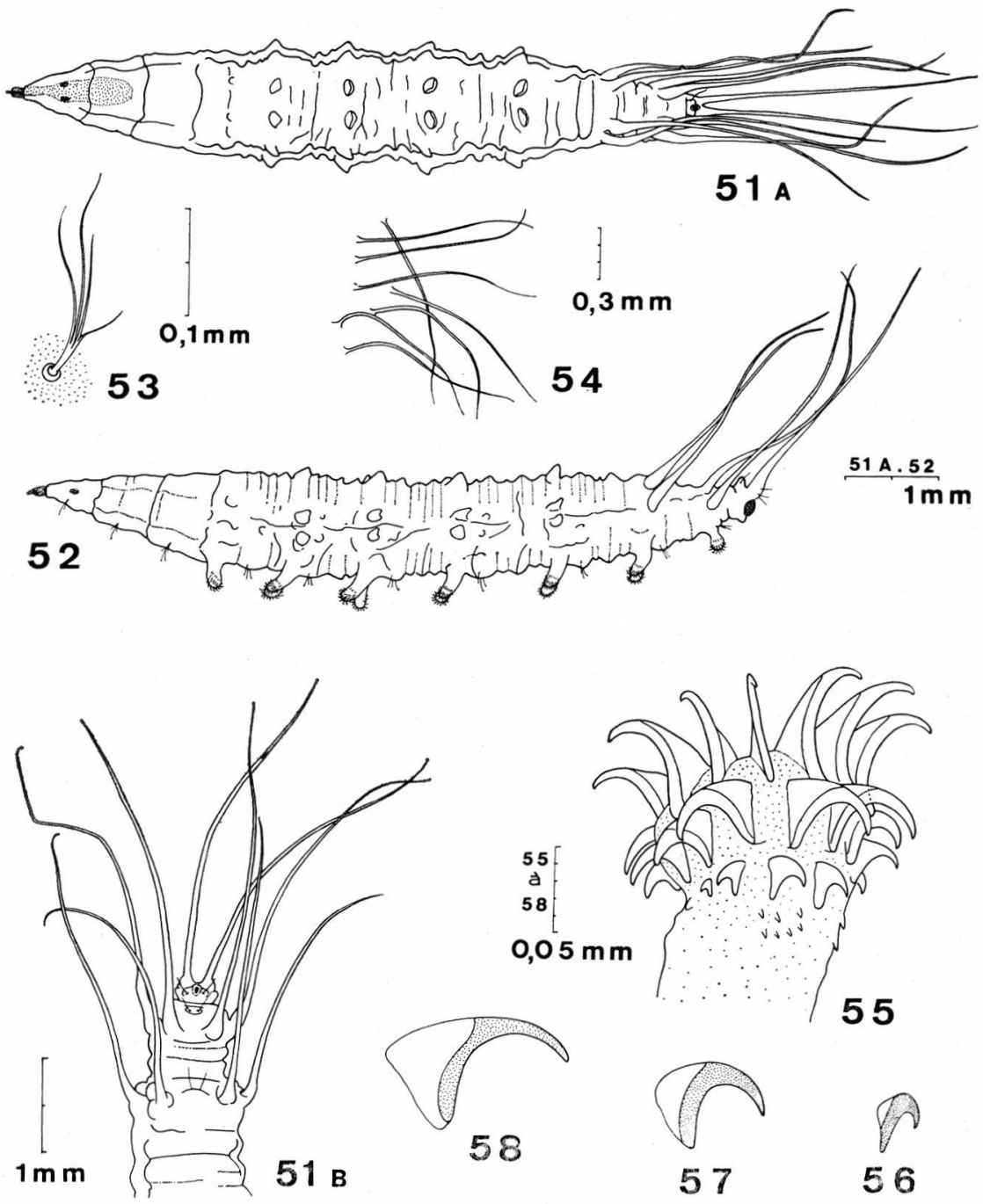

Planche VII : fig. 51 à 58 (larve d'A. crassipes).

- Fig. $51 \mathrm{~A}$ : larve de $10 \mathrm{~mm}$ en vue dorsale; Fig. 52 : la même en vue latérale; Fig. $51 \mathrm{~B}$ : extrémité de l'abdomen d'une larve de $12,5 \mathrm{~mm}$; FIG. 53 : pédichète; FIG. 54: saillies tégumentaires abdominales dorsales; Fig. 55 : extrémité distale du lobe externe d'un pseudopode II de larve de $10 \mathrm{~mm}$; Fig. 56 à 58 : crochets du rang basal (fig. 56), du second rang (fig. 57) et du rang apical (flg. 58). 


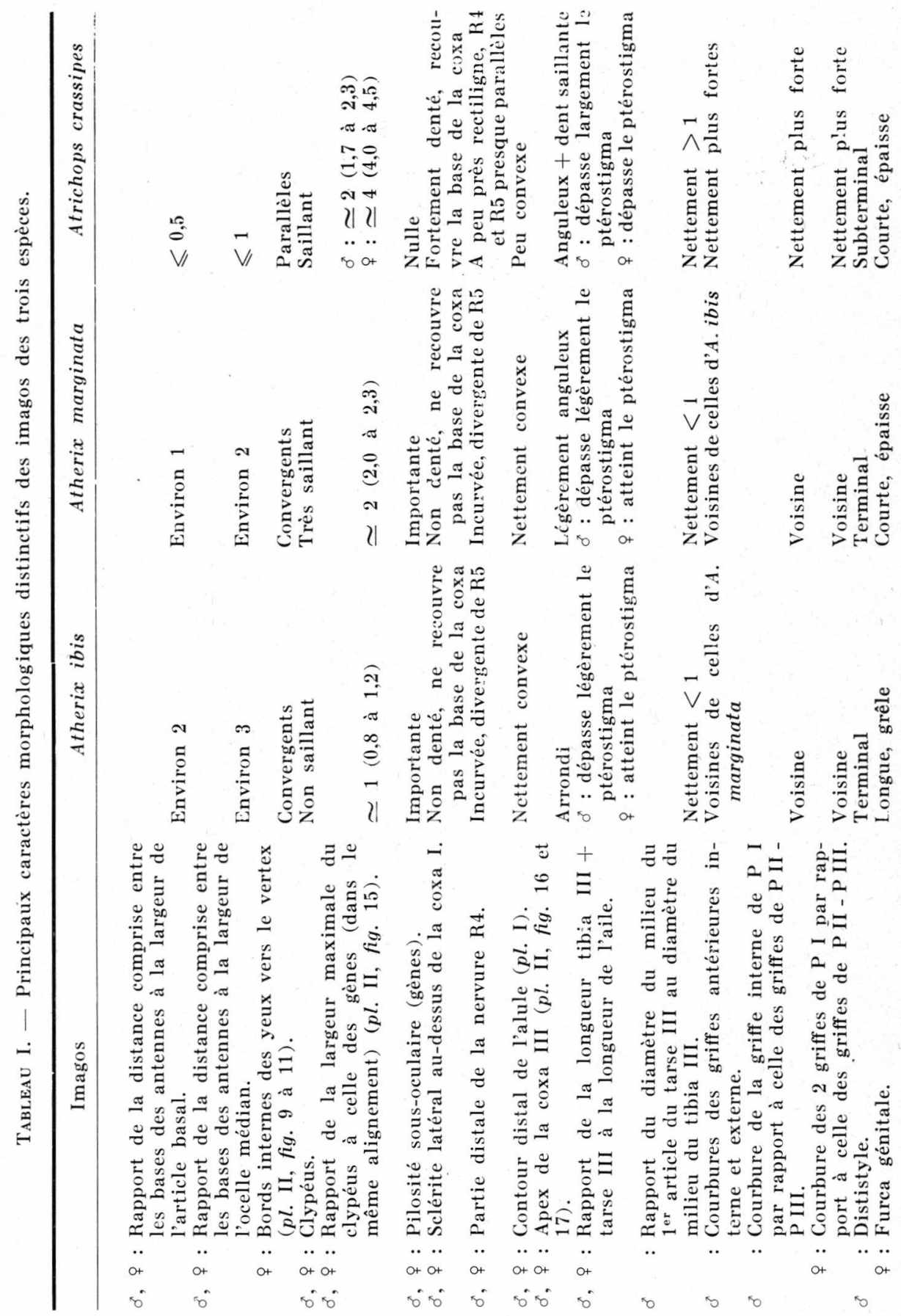




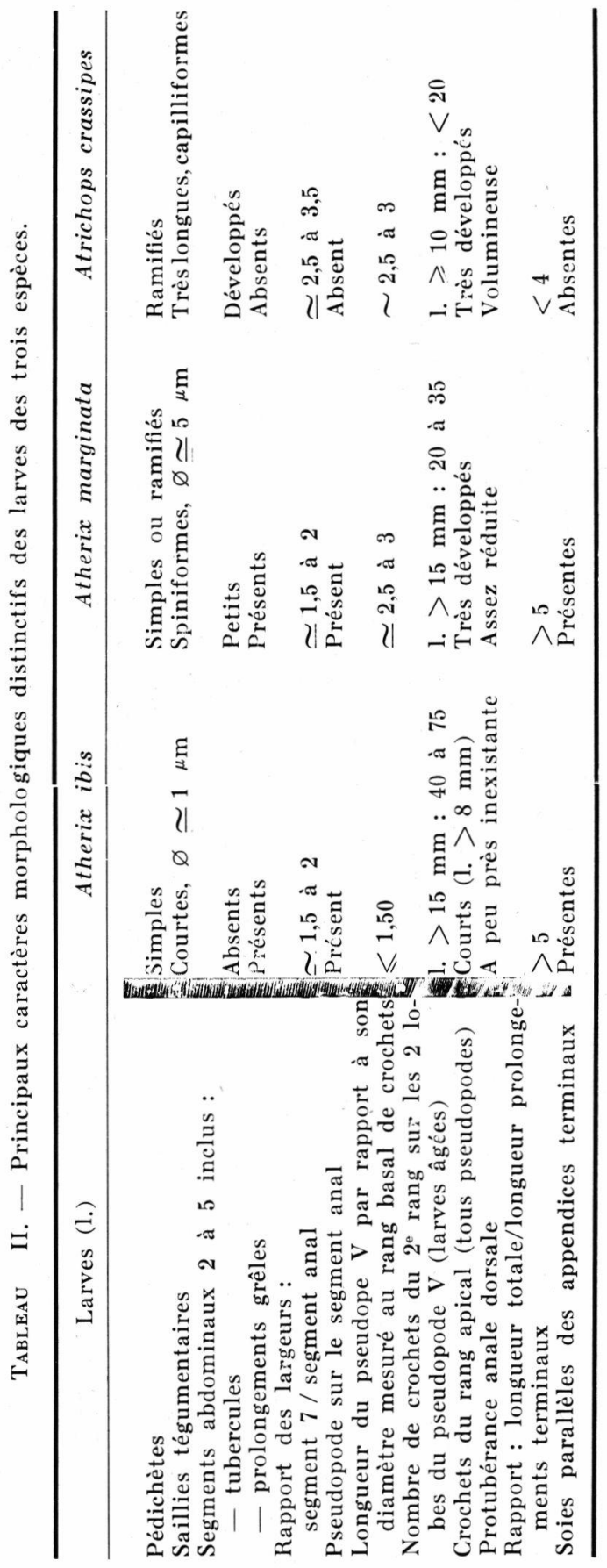


Le Lot à $60 \mathrm{~m}$.

-- Lot : 51.

Le Lot de 80 à $150 \mathrm{~m}$.

- Aveyron : 7 ,

Le Dourdou à $350 \mathrm{~m}$; la Truyère à $220 \mathrm{~m}$; le Lot de 180 à $410 \mathrm{~m}$.

- Lozère : 31 .

Le Lot à $550 \mathrm{~m}$.

Une dizaine de larves d'un ruisseau de l'Entre-Deux-Mers (Gironde) ont également été examinées.

Le tableau II résume les principaux caractères morphologiques des trois espèces. Il montre qu'A. marginata occupe une position intermédiaire entre A. ibis et $A$. crassines.

\section{TRAVAUX CITÉS}

Aldrich (J. M.). 1912. - Flies of the Leptid genus Atherix used as food by California Indians (Dipt.). Ent. News, 23 (4) : 159-163.

Bertrand (H.). 1954. - Les insectes aquatiques d'Europe. Vol. II. Trichoptères, Lépidoptères, Diptères, Hyménoptères. Encycl. ent., Lechevalier, 547 p., Paris.

Borre (P. de). 1879. - Annls Soc. ent. Belg., C. R. du 4-7-1874: 120.

Braukr (F.). 1883. - Die Zweiflügler des kaiserlichen Museums zu Wien. III. Systematische Studien auf Grundlage der Dipteren-Larven nebst einer Zusammenstellung von Beispielen aus der Literatur über dieselben und Beschreibung neuer Formen. Denkschr. Akad. Wiss., Wien, $47: 1-100+5 \mathrm{pl}$.

Brinder (A.). 1961. - Taxonomic Notes on the Larvae of British Diptera, 4. The genus Atherix Meigen (Rhagionidae). Entomologist, $94: 218-220$.

Dufour (L.). 1862. - Consultation sur une larve aquatique. Annls Soc. ent. Fr., $4^{\circ}$ série, $2: 131-138+1$ pl.

Enderi.ein (G.). 1935. - Diptera. In : Die Tierwelt Mitteleuropas, 6, (2) : 259 p., Leipzig.

Frey (R.). 1954. - Studien über ostasiatische Dipteren. III. Rhachiceridac, Rhagionidae, Hilarimorphidae. Notul. ent., 34 (1) : 1-25.

GIARD (A.). 1902. - Note sur la larve de l'Atherix ibis Fabr. (Dipt.). Bull. Soc. ent. Fr. : 220-222.

Greene (C. T.). 1926. - Descriptions of larvae and pupae of two-winged flies belonging to the family Leptidae. Proc. $U . S$. natn. Mus., 70 (2) : $1-20+3 \mathrm{pl}$.

Grünrerg (K.). 1910. — Diptera, Zweiflügler. In Brauer : Die Süsswasserfauna Deutschlands. $2 \mathrm{~A}, 312$ p. Jena.

Hynes (H. B'. N.). 1970. - Ecology of Running Waters. 555 p. Liverpool Universily Press.

IACOB (M.). 1972. - Contributions à la connaissance des représentants de la fam. Rhagionidae (Diptera-Brachycera) de Roumanie. Trav. Mus. Hist. nat. Gr. Antipa, $12: 261-272$.

James (M. T.). 1965. - Family Rhagionidae. In Stone et al. : A Catalog of the Diptera of America north of Mexico. Agric. Handb. Forest Serv. U. S., $276:$ Iv $+1696 \mathrm{p}$.

Johanesen (O.A.). 1935. - Aquatic Diptera. Part II. Orthorrhapha Brachycera and Cyclorrhapha. Mem. Cornell Univ. agric. Exp. Stn, $177: 3-62+12 \mathrm{pl}$.

KaURi (H.). 1967. - Tabanidae et Leptidae. In Iluses : Limnofauna Europaea. xv +474 p. Stuttgart. 
KRrzELJ (S.). 1971. - Diptères Rhagionides de Belgique et d'Europe occidentale. Bull. Inst. $r$. Sci. nat. Belg., 47 (9) : 1-31.

LEONARD (M. D.). 1930. - A revision of the dipterous family Rhagionidae (Lcptidae) in the United States and Canada. Mem. Am. ent. Soc., $7: 1-181$

Lindner (E). 1925. - Rhagionidae. In Lindner : Die Fliegen der Palaearktischen Region. IV, 1, 20:49 p. +2 pl. Stuttgart.

MaCaN (T. T.). 1959. - A guide to freshwater invertcbrate animals. Longmans. 118 p. London.

Marroch (J. R.). 1932. - Notes on exotic Diptera. Stylops, 1 (5) : 112 120.

N.ı.ıтom (A.). 1953. - Descriptions of two new Atherix species from Japan (Diptera, Rhagionidae). Mushi, 25 (3) : 9-12.

Nagitom (A.). 1958. - Studies in the aquatic Snipe-flies of Japan. Part I. Descriptions of the adult (Diptera, Rhagionidae). Mushi, 32 (5) : 47-67.

Nıgıтomi (A.). 1960. - Studies in the aquatic Snipe-flies of Japan. Part II. Descriptions of the eggs (Diptera, Rhagionidae). Mushi, 33 (1) : 1-3.

Nagatom (A.). 1961 a. - Studies in the aquatic Snipe-flies of Japan. Part III. Descriptions of the larvae (Diptera, Rhagionidae). Mushi, 35 (3) : 11-27.

Nagatom (A.). 1961 b. - Studies in the aquatic Snipe-flies of Japan. Part IV. Descriptions of the pupae (Diptera, Rhagionidac). Mushi, 35 (4) : 29-38.

Nig.ıтom (A.). 1962. - Studies in the aquatic Snipe-flies of Japan. Part V. Biological notes. Mushi, 36 (10) : 103-149.

Nigitomi (A.). 1964. - Atherix (Heterosuragina) Nagatomi a new synonym of Atherix (Atrichops) Verrall. Kontyn̂, 32 (1) : 69.

Needham (J. G.). 1903. - Some new life histories of Diptera. In : Aquatic Insects in New York State. Part 4. Bull. N. Y. St. Museum, 68 : $279-287+3$ pl.

Neveu (A.). 1972. - Introduction à l'étude de la faune des Diptères à larves aquatiques d'un ruisseau des Pyrénées-Atlantiques, le Lissuraga. Annls Hydrobiol., 3 (2) : 173-196.

Ordproyo (H.). 1939. - Rhagionidae, Tabanidae, Asilidae, Bombyliidae. In : Ruwenzori Expedition, 1934-35. B. M. (N. H.), $2: 13-47+2$ pl.

Oldroyd (H.). 1964. - The natural history of Flies. 324 p. London.

Oldroyd (H.). 1969. - Diptera Brachycera. Section (a) Tabanoidea and Asiloidea. In : Handbooks for the Identification of British Insects, 9 (4) : 1-128.

Pomvist (E.). 1953. - Studien an Dipterenlarven des Mauerbaches. Wett. Leben, 2 : 165-176.

Rietschei, (P.). 1969. - Les Diptères. In : Grzimeks Tierleben. Traduction française (1972), $2: 381-436+5$ pl. Zurich.

Rozkošný (R.) et SpITzer (K.). 1965 a. - Schnepfenfliegen (Diptera, Rharinnidae) in der Tschechoslowakei. Acta ent, bohemoslov., 62 : 340-368.

Rozkošný (R.) et Spitzer (K.). 1965 b. - Einige Funde von Schnepfenfliegen (Rhagionidae, Diptera) aus Jugoslavien. Acta faun. ent. Mus. Nat. Pragae, 11 : 299-302.

SÉguy (E.). 1926. - Diptères Brachycères. In : Faune de France, 13 : 308 p. Lechevalier, Paris.

Séguy (E.). 1937. - Diptères. In : La Faune de la France, $8: 216$ p. Delagrave, Paris. 
SÉGuy (E.). 1950. - La biologie des Diptères. 609 p. +10 pl. Paris.

SÉguY (E.). 1951. - Ordre des Diptères. In : P. P. Grassé : Traité de Zoologie, $10:$ 449-744. Paris.

SÉGuY (E.). 1955. - Introduction à l'étude biologique et morphologique des Insectes Diptères. Publções Mus, nac. Rio de J. : 1-260.

Strobi (P. (.). . 1898. - Die Dipteren von Steiermark. IV. Mitt. naturiv. Ver. Steierm., $34: 192-298$.

StUckenberg (B. R.). 1960. - Diptera (Brachycera) Rhagionidae. South African animal life. Results of the Lund University Expedition in $1950-1951,7: 216-308$.

Stuckenberg (B. R.). 1965. - The Rhagionidae of Madagascar (Diptera). Ann. Natal Mus., 18 (1) : 89-170.

Stuckenterg (B. R.). 1966. - A new genus and species of Rhagionidae from southern Brazil (Diptera). Proc. R. ent. Soc. London, (B), $35: 57-60$.

Stuckenberg (B.R.). 1973. - The Athericidae, a new family in the lower Brachycera (Diptera). Ann. Natal Mus., 21 (3) : 649-673.

SZILÁDY (Z.). 1932. - 29. Familie : Schnepfenfliegen, Rhagionidae (Leptidae). Tierwelt Dtl., $26: 40-54$. Jena.

SzilAdy (Z.). 1934. - Die Palaearktischen Rhagioniden. Annls nat. Mus. natn. hung., $28: 229-270$.

S.ZILADY (Z.). 1943. - Zwei neue Arten aus der Dipterensammlung von Ignaz Nagy. Folia ent. hung., 8 : 15-17.

Thомas (A.). 1969. - Sur l'importance des Diptères dans l'environnement de quelques cours d'eau des Pyrénées. Annls Limnol., 5 (1) : 61-71.

Tournier (H.). 1874. -- Annls Soc. ent. Belg., C. R., 17 : 89-91.

Walker (F.). 1837 ? -- Dipt. Brit. I. 69, 70 (non vu).

Wesenberg-Iund (C.). 1943. - Biologie der Süsswasserinsekten. 682 p. Copenhagen.

Wirth (W. W.) et Stone (A.) . 1963. - Aquatic Diptera. Family Rhagionidae. In Usinger : Aquatic Insects of California. $508 \mathrm{p}$. Berkeley and Los Angeles. 